\title{
Bioactive Chitosan Nanoparticles and Photodynamic Therapy Inhibit Collagen Degradation in vitro
}

By:

Anousheh Persadmehr

A thesis submitted in conformity with the requirements

for the degree of Master of Science

Graduate Department of Faculty of Dentistry

University of Toronto

(C) Copyright by Anousheh Persadmehr, 2013 


\title{
Bioactive Nanoparticles and Photodynamic Therapy \\ Inhibit Collagen Degradation in vitro
}

\author{
Anousheh Persadmehr \\ Master of Science \\ Faculty of Dentistry \\ University of Toronto
}

2013

\begin{abstract}
This study evaluated the ability of photodynamic therapy (PDT), chitosan nanoparticles (CSnp), or their combination, to inhibit bacterial collagenase-mediated degradation of collagen. Rat type 1 fibrillar collagen matrices were untreated or treated with $2.5 \%$ glutaraldehyde (GD), $2.5 \%$ GD followed by $1 \%$ CSnp, $1 \%$ CSnp, PDT, or $1 \%$ CSnp followed by PDT. Samples, except untreated controls, were exposed to Clostridium histolyticum collagenase. The soluble digestion products were assessed by hydroxyproline assay and the remaining adherent collagen was quantified by picrosirius red (PSR) staining. Collagen treated with CSnp, PDT, or a combination of CSnp and PDT, exhibited less degradation than controls. The abundance of post-treatment residual collagen correlated with the extent of degradation. Fourier transform infrared (FTIR) spectroscopy analysis showed that PDT treatment enhanced collagen cross-linking. Immunoblotting of sedimented CSnp indicated that CSnp and collagenase bound with low affinity. However, CSnp-bound collagenase showed a significant reduction in collagenolytic activity compared with controls.
\end{abstract}




\section{Acknowledgments}

I would like to acknowledge and thank the members of my scientific advisory committee, including Drs. Anil Kishen (primary principal supervisor), Christopher McCulloch (secondary principal supervisor), Calvin Torneck, and Dennis Cvitkovitch. Their

guidance, patience, and support provided me with the perseverance to complete this project. I am truly honored to have had the opportunity to be mentored by such a knowledgeable group.

This project would not have been possible without the help of the Kishen Lab and Matrix Dynamics Group. To my past and present fellow residents, including Drs. Raj Krishan and Pavel Cherkas, a special thank-you for all your support and for making my experience at the faculty memorable and enjoyable.

I would like to extend my gratitude to Dr. Shimon Friedman for giving me the opportunity to be a part of the Graduate Endodontics Program at the University of Toronto. It has been a wonderful three years and I hope to continue to be an active member of this great community.

Most importantly, I would like to thank my amazing family for their endless support in all the decisions that I have made. I am truly blessed to have you in my life.

This study was supported in part by grants from AAE Foundation, CAE Endowment Fund, Alpha Omega Foundation of Canada, and CIHR operating grant to CAM (MOP418228). 


\section{Table of Contents}

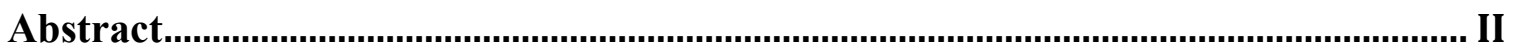

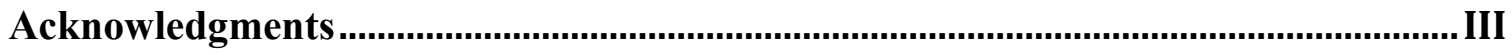

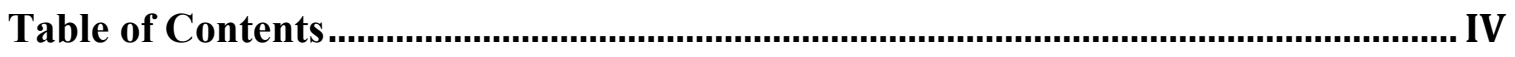

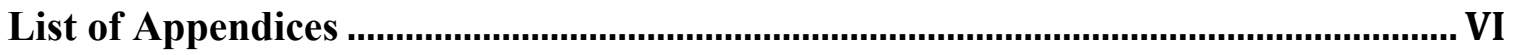

List of Abbreviations................................................................................................... VII

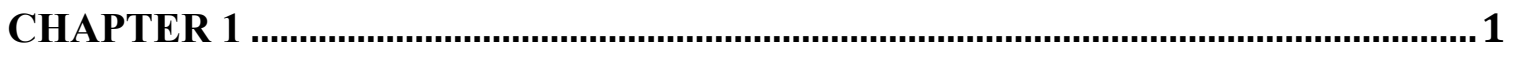

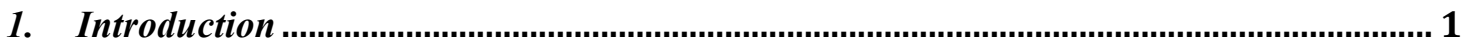

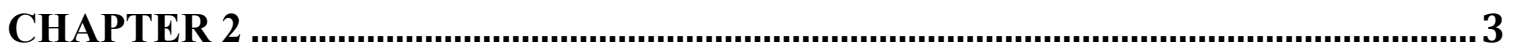

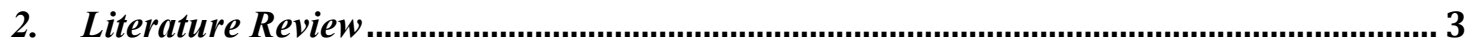

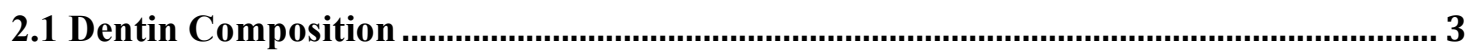

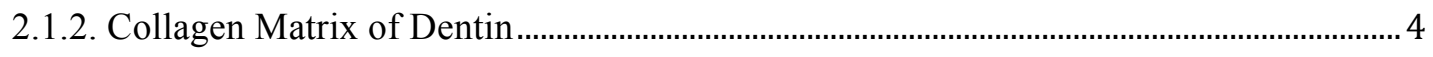

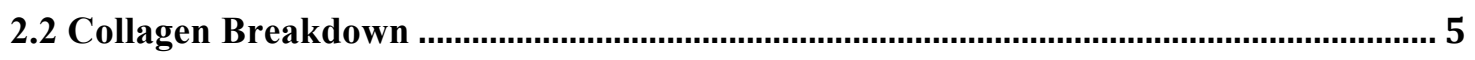

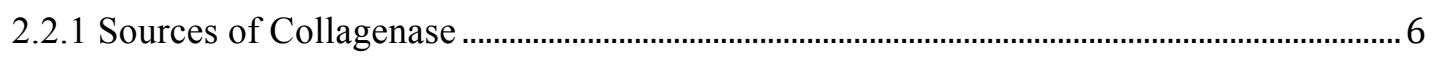

2.2.2 Matrix Metalloproteinases ............................................................................................... 7

2.2.3 Interaction Between Bacteria and Host-Derived MMPs.......................................................... 8

2.3 Ultrastructural Changes to Dentin Following Iatrogenic Procedures............................ 9

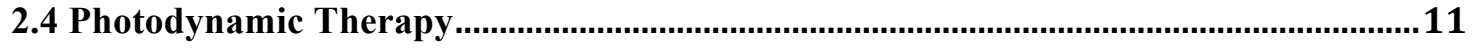

2.4.1 Antimicrobial Effects of Photodynamic Therapy ………………………………............. 13

2.4.2 Photodynamic Therapy in Endodontics.............................................................................. 14

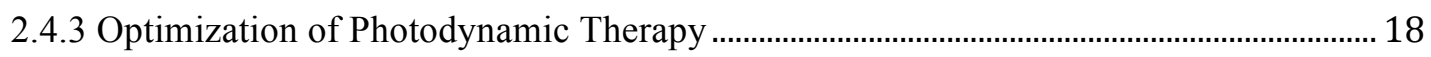

2.5 Strategies to Enhance Chemical and Mechanical Stability of Collagen ........................20

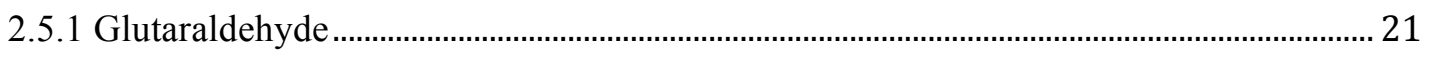

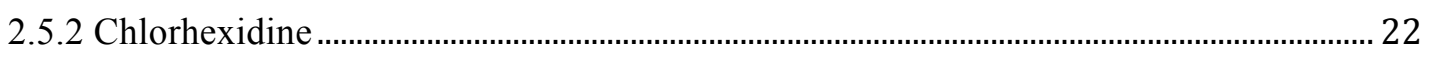

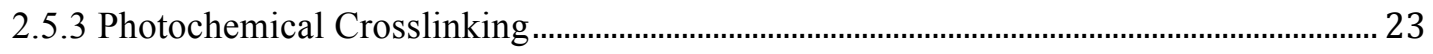

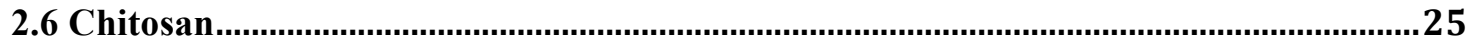

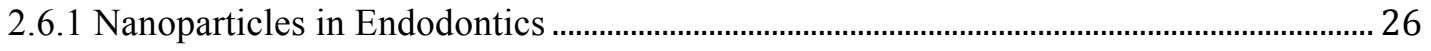

2.6.2 Chitosan Nanoparticles in Endodontics.......................................................................... 28

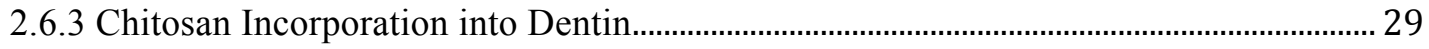




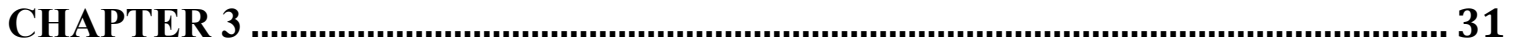

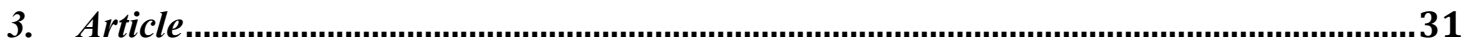

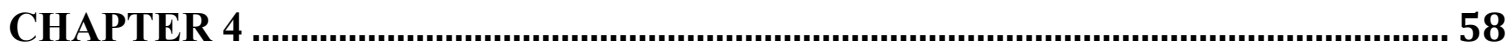

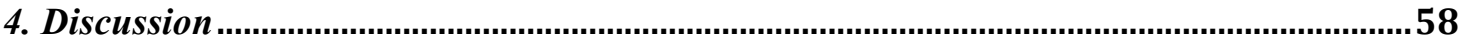

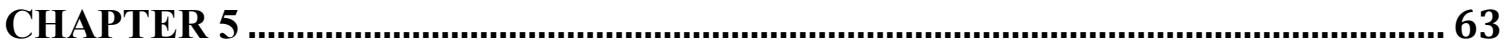

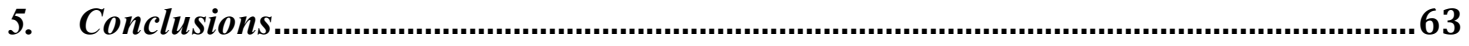

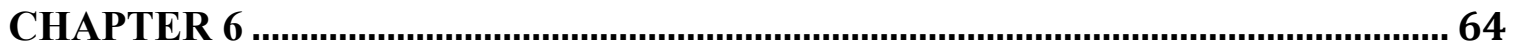

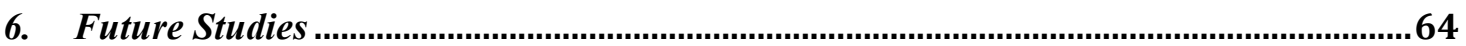

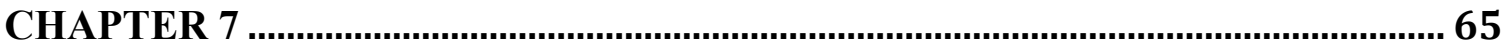

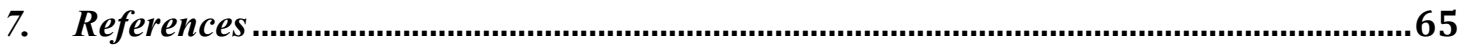

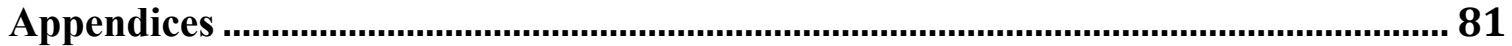

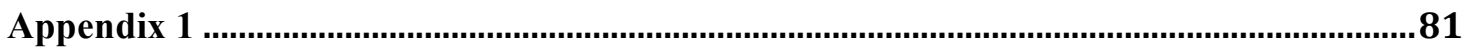

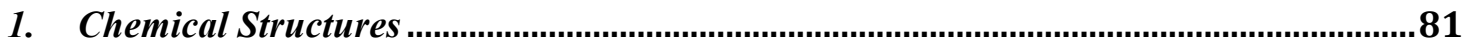

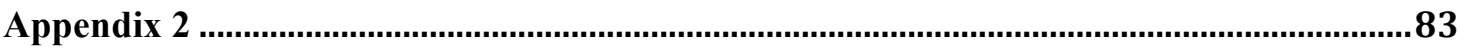

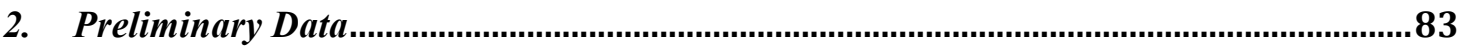




\section{List of Appendices}

\section{Appendix 1. Chemical Structures}

Figure 1. Structure of Collagen

Figure 2. Preparation of Chitosan

Appendix 2. Preliminary Data

Figure 3. Hydroxyproline Assay

Figure 4. Picrosirius Red Stain 


\section{List of Abbreviations}

ACE

AP

BHI

$\mathrm{CaO}$

CHX

CS

CSnp

Cys

DCMs

DI

$\mathbf{E}$

ECM

EDTA

EPI

FTIR

GE

GD

Gly

His

Hyl

LPS

Lys

Mab
Angiotensin-converting enzyme

Apical periodontitis

Brain-heart infusion

Calcium oxide

Chlorhexidine

Chitosan

Chitosan nanoparticles

Cysteine

Demineralized collagen matrices

Deionized water

Modulus of elasticity

Extracellular matrix

Ethylenediaminetetraacetic acid

Efflux pump inhibitors

Fourier transform infrared spectroscopy

Genipin

Glutaraldehyde

Glycine

Histidine

Hydroxylysyl

Lipopolysaccharides

Lysyl

Monoclonal antibody 


\begin{tabular}{|c|c|}
\hline MgO & Magnesium oxide \\
\hline MIX & Mixture of glycerol:ethanol:water \\
\hline MMPs & Matrix metalloproteinases \\
\hline $\mathrm{NaOCl}$ & Sodium hypochlorite \\
\hline $\mathrm{NaOH}$ & Sodium hydroxide \\
\hline OH-Pro & Hydroxyproline \\
\hline PA & Phosphoric acid \\
\hline PA & Proanthocyanidin \\
\hline PBS & Phosphate buffered saline \\
\hline PDT & Photodynamic therapy \\
\hline PEG & Poly-ethylene glycol \\
\hline PEI & Polyethyleneimine \\
\hline PMNs & Polymorphonuclear leukocytes \\
\hline PS & Photosensitizer \\
\hline PSR & Picrosirius red \\
\hline RB & Rose Bengal (photosensitizer) \\
\hline ROS & Reactive oxygen species \\
\hline SEM & Scanning electron microscopy \\
\hline ТВО & Toluidine blue $\mathrm{O}$ \\
\hline TIMPs & Tissue inhibitors of metalloproteinases \\
\hline TiO2 & Titanium dioxide \\
\hline Trp & Tryptophan \\
\hline Tyr & Tyrosine \\
\hline UTS & Ultimate tensile strength \\
\hline $\mathrm{ZnO}$ & Zinc oxide \\
\hline ZnOE & Zinc-oxide eugenol \\
\hline
\end{tabular}




\section{CHAPTER 1}

\section{Introduction}

Primary apical periodontitis (AP) is an expression of pathophysiology induced by the interaction of host tissue with microorganisms and their products present in an infected root canal (1). Effective control of these microorganisms and stabilization of root canal dentin have a significant impact on the long-term survival of root canal treated teeth. Conventionally, such treatment employs a chemo-mechanical approach, that is, a combination of root canal enlargement and shaping with metal instruments and chemical irrigants that disinfect the root canal and prepare the dentin wall in a manner that maximizes sealing of the root canal with a cement and filling core. Nevertheless, currently used protocols have failed to predictably render the root canal system free of infection $(2,3)$ nor have they assured preservation of dentin matrix integrity/stability (4). Persistence of bacteria in the root canal has been identified as a contributing factor to an unfavourable treatment outcome (5-7), while the adverse effects of commonly used root canal irrigants/medicament/sealer on the integrity of dentin matrix collagen may impact the resistance of the root dentin to fracture (8-10).

These limitations highlight the need for additional or alternative treatment strategies that are capable of enhancing the efficacy root canal disinfection and protecting/ strengthening the dentin, to reduce the risk of fracture. One promising method of achieving these goals is the adaption of Photodynamic therapy (PDT) and polycationic 
bioactive nanoparticles as a protocol in root canal preparation. The effectiveness of PDT in root canal disinfection has been demonstrated by in vitro and in vivo studies (11). PDT-generated reactive oxygen species (ROS) increase the number of intermolecular cross-links between adjacent collagen fibrils, which may enhance the resistance of collagen to enzymatic degradation. Since collagen is the principal protein of the dentin matrix, increased collagen cross-linking could improve the mechanical properties of dentin affected by endodontic infection (12).

Dentin can also be reinforced by the incorporation of biopolymers into the collagenassociated fraction of the matrix. Chitosan (CS), a natural hydrophilic polycationic polymer, is markedly biocompatible, exhibits a broad antimicrobial spectrum $(13,14)$ and may increase the resistance to fracture and enzymatic degradation of treated collagen $(12,15,16)$. This thesis aims to evaluate the effects of PDT and chitosan nanoparticles (CSnp) on the resistance of collagen to enzymatic degradation and to further assess the mechanisms involved in this process. The ability to stabilize dentin collagen may serve as a guard against undermining its physical properties and reducing its potential for fracture. 


\section{CHAPTER 2}

\section{Literature Review}

\subsection{Dentin Composition}

Dentin is the main component of the human tooth. It is a mineralized connective tissue (17) consisting of an inorganic and an organic phase. The inorganic or mineral phase, principally hydroxyapatite, comprises approximately $70 \%$ of dentin weight $(45 \%$ of its volume), and provides it with stiffness and elastic modulus (strength) (8). The organic phase of dentin, in the form of a matrix, represents approximately $20 \%$ of its weight (33\% of its volume), and contributes to the ultimate tensile strength and toughness of dentin (8). The remaining fraction is water (17) and its distribution varies approximately 20 -fold from superficial to deep dentin (18). The principal constituent ( $\sim 90 \%)$ of the

organic phase, or matrix, is Type I fibrillar collagen, mostly in the form of a heteropolymer with two $\alpha 1$ chains and one $\alpha 2$ chain (19). There are three associated domains, the $\mathrm{NH}_{2}$-terminal non-triple helical (N-telopeptide), the central triple helical, and the $\mathrm{COOH}$-terminal non-triple helical (C-telopeptide) domains. Proteoglycans and other non-collagenous proteins are distributed between the collagen fibrils and constitute approximately $10 \%$ of the matrix. They play an important role in matrix mineralization. 


\subsubsection{Collagen Matrix of Dentin}

Collagen consists of three left-handed polypeptide helices, stabilized by inter-chain hydrogen bonds (20) (Fig. 1). Disruption of the stabilizing bonds and denaturation of the collagen molecule results in the formation of gelatin (21). Each of the three $\alpha$ polypeptide chains of collagen is more than 1000 residues long, and is characterized by repeating sequences of Gly-X-Y, with glycine, the smallest amino acid, occurring in every third residue. Significant amounts of two amino acids, proline and hydroxyproline (hydroxylated proline), appear in the $\mathrm{X}$ and $\mathrm{Y}$ positions. This triplet-repeat sequence, gives collagen a unique tertiary structure (22).

Collagen matrix is a major contributor to the viscoelasticity, mechanical stability, toughness, and tensile strength of the dentin $(8,23,24)$. The integrity of the collagen is maintained by endogenous covalent intermolecular crosslinking of its three helically arranged collagen fibrils $(25,26)$. Disruption of the cross-linkage undermines collagen stability, and leads to alterations in the physical properties of the dentin. An increase in the number of the crosslinks, on the other hand, has the potential to enhance the mechanical properties and increase dentin resistance to enzymatic degradation (12). 


\subsection{Collagen Breakdown}

Collagenase is an enzyme that cleaves the helical regions of collagen fibril linkage and is involved in collagen degradation. Under normal conditions, this enzyme is produced by fibroblasts and macrophages and plays a role in the maintenance and repair of extracellular matrix. Several proteolytic microorganisms, identified as putative oral/endodontic pathogens, also have the capacity to produce collagenase (27-29). The production of this enzyme allows them to acquire small amino acids derived from collagen degradation of host tissue that are essential for their survival. Since root canal infection is a "biofilm-related disease" in which these microorganisms are found $(30,31)$, they commonly interact with the root canal dentin during the disease process. This bacteria-dentin interaction may be prolonged if the initial disinfection protocol is unsuccessful in eliminating their presence. Prolonged bacterial interaction with dentin can potentially result in the degradation of matrix collagen and altered physical characteristics of dentin. Evidence has also been presented to suggest that there is a local up-regulation of collagenase production during the early phases of root canal infection, by resident fibroblasts and migration of the collagenase-producing cells associated with the host immune-inflammatory response $(28,32)$. To what extent each plays a role in affecting dentin stability is yet to be determined. Previous studies have also identified a family of matrix metalloproteinases (MMPs), endogenous metal containing proteinases, present in the dentin matrix in bound and active forms, as possible contributors to the degradation of collagen during numerous pathological and chemotherapeutic events (33). 


\subsubsection{Sources of Collagenase}

True collagenases cleave helical regions of collagen fibrils at physiological $\mathrm{pH}$ and temperature (21). Mammalian fibroblasts and inflammatory cells, including macrophages and neutrophils, produce collagenases and release these enzymes during the inflammatory response (34). Other mammalian proteases, including pepsin, trypsin, chymotrypsin, and papain can degrade nonhelical regions of collagen and gelatin.

An increasing number of putative oral pathogens have been reported to produce collagendegrading enzymes (21). Collagenase is a potential virulence factor expressed by pathogenic bacteria associated with endodontic disease $(28,35)$. The presence of collagenase gene $p r t C$ was confirmed in Porphyromonas gingivalis (29). Clinical isolates of black-pigmented anaerobes including Porphyromonas asaccharolytica, Porphyromonas endodontalis, Prevotella intermedia, and Prevotella melaninogenica, have been identified as collagenolytic species $(21,36,37)$. Collagenolytic activity of a number of black-pigmented bacteria, isolated from dentoalveolar abscesses has been confirmed (38). Furthermore, a number of other collagenolytic organisms including Bifidobacterium spp., Peptostreptococcus sp., Eubacterium sp., and Peptococcus sp. have been isolated from necrotic pulp chambers (39). Gelatinase enzyme has been isolated from Enterococcus faecalis (40), a bacterium that is commonly associated with persistent endodontic infections. The production of collagen degrading enzymes is a virulence factor that may provide amino acids for bacterial growth and facilitate the spread of 
bacteria (21). Bacterial proteinases are also capable of activating latent mammalian collagenases and contribute indirectly to collagen degradation (41).

\subsubsection{Matrix Metalloproteinases}

Matrix Metalloproteinases (MMPs), also called matrixins, form a multigene family of zinc-dependent endopeptidases that mediate the degradation extracellular matrix (ECM) molecules, including native and denatured collagen (42). MMPs are synthesized and secreted as proenzymes and are activated in the extracellular matrix. Their proteolytic activity is regulated by the tissue inhibitors of metalloproteinases (TIMPs) and by the nonspecific inhibitors such as $\alpha 2$-macroglobulin $(43,44)$. MMPs contribute to both physiological and pathological tissue remodelling. The synthesis and organization of extracellular matrices precedes mineralization in the morphogenesis of dental hard tissue. Upon mineralization, MMPs may be trapped in the mineralized phase bound to matrix components or to hydroxyapatite crystals. Previous studies have detected MMP-2 (45, 46), MMP-8 (46, 47), MMP-9 (46) and MMP-20 (48) in mature mineralized dentin. Characteristics of the degradation products can distinguish bacterial and mammalian collagenases. Bacterial collagenases digest the collagen substrate into small peptide fragments. In contrast, mammalian collagenases cleave the collagen at a single site, resulting in classic $1 / 4$ and $3 / 4$ segments (49). 


\subsubsection{Interaction Between Bacteria and Host-Derived MMPs}

Bacteria and their products may directly stimulate MMP activation. It has been suggested that MMPs participate in caries progression. In carious lesions, microbial enzymes/acids result in demineralization, accompanied by dentin organic matrix degradation. Recent studies have evaluated the contribution of host-derived MMPs to the breakdown of collagen matrices, observed in the pathogenesis of dentin caries (50-52). This was supported by the findings that the $\mathrm{pH}$ changes taking place in caries lesions are powerful activators of MMPs and was further supported by an in vivo study in which the MMP inhibition significantly down-regulated dentinal caries lesion progression (48). Sato et al. (46) reported that sonic bacterial extracts from Porphyromonas gingivalis, an endodontic pathogen, activate gelatinase A (MMP-2) and inactivate tissue inhibitor of metalloproteinases (TIMP-1 and TIMP-2), leading to the breakdown of the connective tissue. In a study by Reynaud af Geijersstam et al. (53) one of the tested E. faecalis strains stimulated MMP-8 release from human polymorphonuclear leukocytes (PMN). These findings can be extrapolated to residual intracanal bacteria and their effect on activation of exposed MMPs and TIMPs in partially demineralized dentin following endodontic treatment. Vier et al. (54) reported the occurrence of apical internal root resorption in $74.7 \%$ of roots in endodontically involved teeth. These resorption spaces are located short of the limit of apical instrumentation. Thus, standard cleaning and shaping procedures will not successfully eliminate bacteria in this region, which remain in the spaces created by dentin resorption. The residual bacteria may activate dentinal MMPs, contributing to the breakdown of demineralized collagen matrices and weakened dentin 
structure. MMPs, as collagenase and gelatinase have been found in gingival crevicular fluid and saliva (55-58). Given the various sources of collagenase, a non-specific collagenase inhibitor with high inhibition, physico-chemical characteristic to adhere to inherently moistened dentin, and with substantivity is desired.

\subsection{Ultrastructural Changes to Dentin Following Iatrogenic Procedures}

Structural changes in dentin as a result of chemicals used for endodontic irrigation have been extensively studied in the literature $(9,10,59-62)$. Changes in the organic and inorganic components of dentin will affect the mechanical properties of dentin. Various

concentrations of chelating agents, including ethylenediaminetetraacetic acid (EDTA) and citric acid, can reduce dentin microhardness $(60,61,63)$. Sodium hypochlorite $(\mathrm{NaOCl})$ is the most commonly used endodontic antimicrobial irrigant, which has the ability to dissolve the organic material. The use of $5.25 \% \mathrm{NaOCl}$ has been reported to decrease Young's modulus of dentin, in addition to its flexural stress (62). Reduction in dentin elasticity after treatment with $\mathrm{NaOCl}$ has also been reported $(60,61,63)$. This may be due to the dissolution of the organic component of the dentin by $\mathrm{NaOCl}$.

Dentin demineralization associated with environmental (e.g., acidic luting cements) or pathological (e.g., bacteria mediated) processes may result in the release of MMPs. These exposed MMPs may be activated by low $\mathrm{pH}$ and participate in the degradation process of 
demineralized dentinal matrices (50). Various restorative procedures and irrigation procedures result in the demineralization of dentin. Ferrari et al. (64) examined demineralized collagen matrices (DCMs) created by acidic zinc phosphate cement over time. Scanning electron microscopy revealed a progressive degradation of the DCMs, resulting in the loss of density, and structural integrity after 6 to 9 years, while partially disappearing after 10 to 12 years. Furthermore, transmission electron microscopy revealed evidence of collagenolytic activity within DCMs, with loss of cross-banding and unraveling into microfibrils, and gelatinolytic activity that resulted in disintegration of the microfibrils. The authors speculated bacterial colonization and the release of bacterial enzymes and of host-derived MMPs to have contributed to the degradation of collagen matrices in root dentin. The application of etch-and-rinse and self-etch adhesives in dentin bonding techniques have also been shown to activate collagenolytic and gelatinolytic processes mediated by the activation of endogenous MMPs in coronal dentin $(65,66)$. Latent MMPs bound to dentin matrix are exposed following etching procedures and the low $\mathrm{pH}$ activates the enzymes by inducing conformational changes and exposing the catalytic domain (50). This enzymatic degradation may be detrimental to intraradicular dentin with the recent widespread use of bonded fiber posts and resinbased root filling materials. Collagen degradation mechanisms may also occur in the root canal system following smear layer removal with EDTA, a commonly used endodontic irrigant. In an in vitro study, Carrilho et al. (33) demonstrated collagen solubilization and decrease in the modulus of elasticity (E) following demineralization with either EDTA or phosphoric acid (PA) and incubation in artificial saliva. Given that collagen matrix is related to the fracture toughness and tensile strength of dentin (33), disinfection of root 
canal dentin with current chemicals may lead to weakening of dentin structure. Therefore, treatment strategies to inhibit the release and to resist the potential of collagen degradation by MMPs would be compelling in endodontics.

In summary, the bacterial/host derived enzymes and iatrogenic chemicals can mediate collagenolytic activity on root dentin. This change can compromise the mechanical integrity of dentin, potentiate fracture propagation in dentin, and breakdown canal wall and root filling interface. All the above may lead to treatment failure and eventual tooth loss. The crosslinking of demineralized collagen may improve the mechanical/interfacial integrity, which may in turn hinder bacterial ingress and re-colonization within endodontically treated teeth.

\subsection{Photodynamic Therapy}

Photodynamic therapy (PDT) uses a specific wavelength of light to activate a light compatible nontoxic dye known as a photosensitizer (PS). Following the absorption of a photon, PS changes from a ground state $\left(S_{0}\right)$ to a first excited state $\left(S_{1}\right)$. In its excited state, PS may return to a ground state or pass into a triple excited state $\left(T_{1}\right)$ by intersystem crossing. In its triple state, PS is highly reactive and can produce cell injury via two different pathways. In the Type I pathway, T1 PS reacts directly with the target cell through a transfer of hydrogen or an electron. This forms radical ions that can react further with oxygen, to produce cytotoxic species such as superoxide anion, hydrogen 
peroxide, and lipid-derived radicals that are toxic to the target cell. The Type II pathway involves transfer of energy from the T1 PS to available ambient oxygen, giving rise to the formation of a highly reactive singlet-oxygen $\left({ }^{1} \mathrm{O}_{2}\right)$ that is equally toxic $(11,67)$. PDT may be a novel approach of overcoming several of the limitations identified in conventional root canal treatment.

Preliminary studies have demonstrated that PDT in the presence of a biopolymer, chitosan can protect the matrix collagen against the action of collagenase, and at the same time, destroy microorganisms, particularly those in biofilm, in the root canal. Singletoxygen and other free radicals that are formed during PDT kill microorganisms by disrupting essential microbial cell molecules, including cytosolic and membrane proteins, membrane lipids, and nucleic acids (68-72). As an example, the application of PDT using methylene blue as a PS against E. faecalis, has been shown to functionally impair the cell wall, damage chromosomal DNA, and degrade membrane proteins (72). PDT also has been reported to break single, as well as double-stranded DNA, resulting in the disappearance of the plasmid super-coiled fraction often associated with antibiotic resistance $(73,74)$. Singlet oxygen has a lifetime of less than $0.04 \mu \mathrm{sec}$, and a radius of action of less than $0.02 \mu \mathrm{m}$ in a biological environment (75). As a strong oxidizing agent singlet oxygen may also react with photo-oxidizable amino acid residues such as His, Cys, Trp, and Tyr to produce a reactive protein species. This activated protein may, in turn, interact with free amino groups in another protein to form cross-links (76). 


\subsubsection{Antimicrobial Effects of Photodynamic Therapy}

The antimicrobial efficacy of PDT has been shown to be dependent upon the target microorganism. Gram-positive and Gram-negative bacteria have been reported to have a different susceptibility to PDT $(77,78)$. Gram-positive species have a porous cell membrane composed of peptidoglycan and lipoteichoic acid surrounding a cytoplasmic membrane and allows easy passage of neutral, anionic, or cationic PSs into the cell. In contrast, Gram-negative bacteria have an outer membrane rich in lipopolysaccharides (LPS), a thin intermediate peptidoglycans layer, and an inner cytoplasmic membrane (79) that strictly regulates movement of molecules into the cell. Consequently, only cationic PS molecules are able to pass through the membrane and penetrate the cell. Thus, negatively charged antimicrobial PS have limited application in the treatment of polymicrobial infections such as those found in the root canal, while positively charged PSs such as porphyrins, phthalocyanines, and phenothiazines (e.g. toluidine blue $\mathrm{O}$ (TBO) and methylene blue) are generally more effective photosensitizer $(80,81)$.

Soaps, detergents, and many of the dyes used in PDT are exogenous amphiphilic substances, having both hydrophilic and lipophilic properties. Bacteria and mammalian cells have proteins present in their cell wall that act as an efflux pump to rid the cell of potentially harmful amphiphilic molecules (82). This process confers a degree of resistance to PDT, as well as other antimicrobial protocols and limits the antimicrobial action of PDT, especially in microorganisms found in biofilm $(83,84)$. Phenothaizinium, a commonly used PSs, which includes methylene blue and its analogues, are classified as 
amphipathic cations and have been reported to be substrates of bacterial multi-drug efflux pumps (85). Recently, agents known as efflux pump inhibitors (EPI) have been studied as a means of overcoming this mode of bacterial resistance (86). One such study was reported by Kishen et al. (87) who demonstrated an increased disinfection potential against E. faecalis biofilm when an EPI was combined with phenothiazinium PSs for PDT disinfection. Interestingly, the addition of an EPI to phenothiazinium PSs did not influence the anti-biofilm effect of chitosan nanoparticles (88).

\subsubsection{Photodynamic Therapy in Endodontics}

The antimicrobial effects of PDT on endodontic pathogens have been investigated in a number of studies. Fimble et al. (89) investigated the photodynamic effect of a methylene blue PS on a multispecies biofilm grown in the root canal of previously extracted human teeth and recorded a kill rate of approximately $80 \%$. They concluded PDT may have potential as an adjunct in root canal disinfection following the use of a standard chemomechanical protocol. The type and formulation of the PS used in PDT has also been shown to influence its antimicrobial potential (72). Adding an emulsion of oxidizer and oxygen carrier to methylene blue, for example, was shown to enhance its antimicrobial effect against E. faecalis (90) and the use of a methylene blue PS dissolved in a mixture of glycerol, ethanol, and water in the presence of an irradiation medium (perfluorodecahydro-napthalene) proved successful in eliminating E. faecalis biofilm in the root canal of an extracted human tooth model (91). 
The use of PDT as an antimicrobial protocol in endodontics has been reported in the literature, particularly in regards to its effect on E. faecalis, a fastidious Gram-positive facultative anaerobe implicated in post-treatment disease (92). Several years later, Soukos et al. (93) published a study highlighting the antimicrobial effect of PDT using methylene blue as PS on a variety of endodontic pathogens. Not surprisingly, he reported complete elimination of all bacteria, with the exception of E. faecalis, which only displayed a $53 \%$ reduction. That same year, Williams et al. (94) reported the effective use of PDT using TBO as a PS and a red light source against a variety of Gram positive and Gram negative endodontic pathogens that included $S$. intermedius, $P$. micros, $P$. intermedia, and $F$. nucleatum. Later in 2008 and 2009, George and Kishen (95) and Meira et al. (96) also published reports highlighting the effective killing of E. faecalis with PDT using both methylene blue and TBO. George and Kishen (95) reported that methylene blue modified with a mixture of glycerol:ethanol:water (MIX) could effectively penetrate dentinal tubules, enhance singlet oxygen generation, and produce a pronounced antibacterial effect. In 2008, they showed that PS formulation, which incorporated an oxidizer and an oxygen carrier in the form of an emulsion, was effective against $E$. faecalis even when present as a mature endodontic biofilm (90). However, despite its promise as an effective antimicrobial agent in in vitro studies, PDT has yet to overcome several of the obstacles that limit its use clinically.

The complex anatomy of the root canal system, particularly in multi-rooted teeth, poses a challenge to the application of PDT in clinical endodontics. It limits distribution of PS in therapeutic concentrations of PS to all parts of the root canal system, especially the cul de 
sacs, and isthmuses where biofilm is often present, it hampers the penetration of optimum light energy to these same area, it requires an adequate supply of oxygen which is limited in the deeper and recessed regions of the root canal system, and finally, it has a potential to cause dentin discolouration especially when certain dyes are used as the PS (11).

Despite these limitations, some studies have supported the use of PDT in clinical endodontics. In 2006, Bonsor et al. (97) evaluated the antimicrobial efficacy of PDT using TBO as a PS and a diode laser light source as a supplement to what they described as "standard root canal enlargement and disinfection". The combined protocols yielded a $10 \%$ increase in bacterial killing, when compared to cleaning and shaping alone. In 2008, Garcez et al. (98) used a PDT disinfection protocol employing polyethyleneimine (PEI) chlorin (e6[ce6]) conjugate repeated during two-appointments and concluded that the second session proved to be more effective than the first despite the fact that there was no significant reduction in bacterial load. Garcez et al. (99) also studied the effect of combined PDT and endodontic treatment in the teeth of patients with root canals containing bacteria that had proven to be resistant to antibiotics that had been used as a root canal dressing. They reported that the combined protocols were effective in eliminating the drug-resistant species and rendered all of the root canals bacteria-free. Accordingly, these last two studies designated PDT root canal disinfection as a supplement to chemomechanical disinfection and not a primary one. But even in this role it has been stated that additional research is warranted in order to improve anti-biofilm efficacy of PDT by optimizing the PS formulation and maximizing the light delivery to all regions of the root canal system (11). 
The commonly used irrigants in root canal disinfection are potentially harmful to both host tissue and the dentin substructure (100). PDT, on the other hand, can be designed to be more target specific, that is lethal to target microorganisms without producing an adverse effect on host tissue. This has been reported to occur at the photosensitization periods and light fluences required for effective disinfection of the root canal in a number of studies. PDT is characterized by dual selectivity: specific binding to a target microorganism and constraint irradiation to a specific volume. Currently, methylene blue, toluidine blue $\mathrm{O}$, rose bengal, erythrosine, chlorin (e6), and hematoporphyrin are being investigated as PSs for use in clinical practice (11). Soukos et al. (101) have reported that under the protocol they had used experimentally, which used toluidine O as a PS and red light as a light source, they were able to effectively kill a test organism, S. sanguis, without causing significant injury to their test cells, human gingival keratinocytes and fibroblasts. In a similar study, using methylene blue as the PS and red light as the light source, Xu et al. (102) reported that it was possible to inactivate selected endodontic pathogens without producing significant injury to human gingival fibroblasts and osteoblasts. Using methylene blue-mediated PDT, different results were reported by Soncin et al. (103) and George and Kishen (95). Soncin et al. (103) reported selective (6:4 ratio) killing of $S$. aureus over human fibroblasts and keratinocytes with cationic pthalocyanine and low light fluence. Furthermore, George and Kishen (95) reported a $97.7 \%$ reduction of E. faecalis and a 30\% reduction in the viability of human fibroblasts when methylene blue was used as the PS and red light as the light source. One source of this toxicity may be the type of PS employed. 


\subsubsection{Optimization of Photodynamic Therapy}

Photosensitizers are chemical agents and when used in high concentrations may produce toxic effects clinically. Furthermore, the toxicity of the breakdown products of PSs, particularly those that have been suggested for clinical use, have not been fully identified and categorized. Until such information becomes available, caution in the use of PDT as a principle approach to root canal disinfection should be exercised (11). PSs are more effective agents for PDT when they are prepared in a monomeric form suspended in a suitable carrier. If caution is not exercised in their preparation the clinical effectiveness of the PS can be compromised. PS prepared in an aqueous environment, for example, may co-aggregate resulting in a self-quenching effect that reduces the yield of singlet oxygen and its antimicrobial effect (104). This effect was investigated by George and Kishen (105) who demonstrated an increased aggregation and a diminished antimicrobial effect of methylene blue PS prepared in water as compared to methylene blue prepared in $70 \%$ glycerol, 70\% poly-ethylene glycol (PEG), and a mixture of glycerol:ethanol:water (MIX). A similar decease in antimicrobial action was also reported by Fimple et al. (89) when Brain-Heart Infusion (BHI) broth was used in the preparation of the PS. The reduced cytotoxic effect in this study was attributed to the presence of environmental serum proteins. In most studies discussed in this review the PSs were prepared in deionized water (DI) or phosphate buffered saline (PBS).

To be effective, the wavelength of the light used in PDT should be specific for the PS selected. This light may be coherent (e.g. lasers) or non-coherent (e.g. lamps). Laser light 
is a highly energized collimated monochromatic beam whereas lamplights are noncollimated, polychromatic and less intense. The use of laser light in this instance is different than, and hence should not be confused with, the use of lasers alone for root canal disinfection. For PDT in root canal treatment, the light must be delivered through a probe attached to a fiber optic cable. Despite all the studies reported thus far, the literature has failed to demonstrate an advantage of one light source over another (11) and the type of light that is best suited in fulfilling the results that are expected.

It is apparent, however, that the strategies that can enhance oxygen concentration in root canal, and optimize the delivery of the PS and light to all of the anatomical complexities of the root canal, including the dentinal tubules and anatomical complexities in the root canal, will prove to be the most suitable for clinical use of PDT in endodontics. Anticipating the use of PDT as a disinfection protocol in root canal treatment, investigators have looked for ways to enhance its effectiveness and at the same time, minimize its collateral damage. They have found that one way of achieving this goal was through the conjugation of PS to a variety of chemical agents. Soukos et al. (106) for example, conjugated chlorin (e6) and a poly-l-lysine chain to methylene blue and reported that the combination was effective in eliminating both Gram-positive and Gramnegative species. Similar findings were reported by Hamblin et al. (107). Rose bengal PS covalently bonded to small polystyrene beads was demonstrated by Bezman et al. to be effective in killing E. coli (108) and PS bound to a monoclonal antibody (Mab), specific to the cell surface antigens expressed by $P$. aeruginosa, was shown to be effective in destroying this microorganism following light activation (109). Gross et al. (110) 
conjugated a PS called bacterio-chlorophyll (Bchl) to rabbit immunoglobulin G (IgG) with high specificity for protein A moieties on cell wall of $S$. aureus and found it significantly improved the kill rate. More recently, the conjugation of nanoparticles with photosensitizer has shown promise. Nanomaterials, such as $\mathrm{TiO} 2, \mathrm{ZnO}$, and fullerenes and their derivatives, have demonstrated a potential to generate singlet oxygen (11) and Klepac-Ceraj et al. (111) and Pagonis et al. (112) have shown a greater killing capacity of E. faecalis in planktonic and biofilm form by methylene blue loaded with poly(lacticco-glycolic) (PLGA) nanoparticles $(111,112)$. The future use of nanoparticles appears bright because they can be conjugated with other agents to further enhance their spectrum of use.

\subsection{Strategies to Enhance Chemical and Mechanical Stability of Collagen}

Collagen is strengthened by native crosslinks. These crosslinks in collagen, in addition to improving its mechanical properties, provide it with greater fibrillar resistance against enzymatic degradation. To increase the inter- and intra-molecular collagen crosslinks and thereby improve the mechanical and biological properties of collagen, several synthetic and natural chemical crosslinkers have been studied $(113,114)$. 


\subsubsection{Glutaraldehyde}

Glutaraldehyde (GD) is a commonly used fixative and it was used as a control in this study. Previous reports have shown the reduced degradation and improved mechanical properties of biological tissues following treatment with GD $(115,116)$. The aldehyde residue on GD reacts with the amino groups of lysyl (Lys) or hydroxylysyl (Hyl) residues, resulting in a network of crosslinks within the biological tissue (115). The treatment of demineralized bovine dentin with GD resulted in a reduction in the free Lys and $\mathrm{Hyl}$ residues in collagen, representing increase in the number of crosslinks (117). The disadvantages of GD as a crosslinking agent include its high cytotoxicity (113) and long treatment time of more than 6 hours, which limits its clinical applicability. Furthermore, Shrestha et al. (12) have found that GD treatment increases UTS of dentin but decreases its toughness, making it more brittle.

Bedran-Russo et al. (114) treated demineralized dentin sections with 5\% GD, 0.5\% proanthocyanidin PBS solution (PA), and $0.625 \%$ genipin PBS solution (GE) for 4 and 40 hours. Proanthocyanidins are naturally occurring bioflavonoids from plant metabolites and are extracted from fruits, vegetables, nuts, seeds and flowers (113). GE is obtained from genoposide, which is isolated from the fruits of Gardenia jasminoides Ellis (115). They found a statistically significant increase in ultimate tensile strength for demineralized dentin after PA and GE dentin treatment, while GD showed no statistically significant difference. The authors speculated that the GD-induced collagen crosslinking in dentin was not sufficient to increase the UTS values. The treatment times tested in this 
study are not clinically practical. In another study, GD treatment was effective in increasing the elastic modulus of demineralized dentin specimens and this increase was limited by the availability of free Lys or Hyl amino groups (118). Treatment of demineralized human dentin with tannic acid, a naturally occurring polyphenol, demonstrated increased stiffness and increased resistance to collagenase degradation in vitro (119).

\subsubsection{Chlorhexidine}

Chlorhexidine $(\mathrm{CHX})$ is a broad-spectrum cationic antimicrobial agent and has been studied as a protease inhibitor in adhesive dentistry to preserve the integrity of collagen matrices in the hybrid layer $(33,120,121)$. In the absence of bacterial and salivary protease, residual collagenolytic activity was found in partially demineralized dentin powder from extracted, caries free teeth $(65,66,122,123)$. CHX has been shown to inhibit MMP-2, -8 , and -9 activities at low concentrations (124). The collagenase activity is inhibited as long as $\mathrm{CHX}$ remains bound to the collagen matrix and CHX-treated bonded dentin was shown to be stable over 14 months (121). To improve the water solubility of $\mathrm{CHX}$, it is used as a salt by the addition of an acid. However, some of the collagenase inhibiting effects is lost in the salt form of CHX (125). When used in a low concentration, Zheng et al. (125) showed that CHX was unable to sufficiently inhibit the activity of collagenase at high concentration. Captopril, a synthetic non-peptidic angiotensin-converting enzyme (ACE) inhibitor, has also been studied as a collagenase 
inhibitor with favourable results (125). Further research is needed to assess its applicability in a clinical setting.

The use of a CHX rinse after irrigation with a demineralizing agent during root canal treatment appears to be one way of controlling the endogenous collagenase activity (120). Gendron et al. (124) demonstrated that calcium chloride prevents the inhibition of endogenous collagenase activity by CHX suggesting that this effect is related to a cationchelating inhibition mechanism.

Previous studies have shown the formation of an orange-brown precipitate when CHX is used in combination with $\mathrm{NaOCl}(126-129)$. This solid precipitate produced may occlude the dentinal tubules and form a barrier between the filling material and dentin surface and therefore increase the risk of microleakage $(127,130)$. This may be a limitation of using CHX rinse for controlling endogenous collagenase activity in endodontic treatment.

\subsubsection{Photochemical Crosslinking}

Another method of stabilizing collagen during root canal treatment is the use of agents that increase collagen crosslinkage. This method provides an additional advantage of increasing the strength of the dentin and its modulus of elasticity $(23,114)$. The highly reactive oxygen species produced during PDT display an ability to interact with the surrounding amino acids to promote crosslinking of collagen fibrils (131-133). The 
increase in the number of intermolecular collagen bonds formed in this process has been identified as a method of increasing the resistance of dentin collagen to degradation that can occur during certain pathological conditions such as caries. Ultimately this may preserve, and perhaps improve, the mechanical properties of the dentin undermined by the infection $(12,114,134)$.

Physicochemical properties of collagen have been modified with the use of photodynamic therapy (131). In this process, photosensitizer such as rose Bengal, is applied to the collagen gel and is activated by absorbing photons from a light with a specific wavelength. Rose Bengal, the photosensitizer used in this study, is anionic and hydrophobic with high quantum yield for photoproducts (135). The resultant photoproducts such as reactive oxygen species react with the surrounding amino acids, forming photochemical crosslinks. In this technique the use of toxic compounds is avoided. In a study by Chan et al. (136) the photochemical crosslinking procedure produced collagen scaffolds with fine microstructures, which strengthened, stiffened, and stabilized the collagen membranes and modified their swelling ratio. 


\subsection{Chitosan}

Chitosan (CS) (poly(1,4),-b-D-glucopyranosamine), is an abundant natural positively charged, hydrophilic bipolymer (13). It contains more than 5000 glucosamine units and is derived from alkaline deacetylation of chitin that is obtained from the shells of crustaceans (Fig 2.) and is available in a variety of physical forms: film, fiber, bead, powder, or as nanoparticles (137). Chitosan is insoluble in most solvents, including water, but is soluble in organic acids such as acetic acid and lactic acid. In addition, CS contains high nitrogen content (6.89\%), making it a useful chelating agent (13).

Chitosan is non-toxic, antimicrobial and antifungal, and acts as a bio-adhesive that attaches to a negatively charged surface (100). The specific mechanism responsible for the antimicrobial action of chitin, chitosan, and their derivatives is still unknown. One proposed mechanisms for its antimicrobial activity is an electrostatic interaction between positively charged CS molecules and the negatively charged surface of a microbial cell that causes the leakage of proteins and other intracellular constituents from the cytosol, eventually leading to cell death $(13,14)$. It has also been speculated that CS may also act as a chelating agent, it can selectively bind to trace metals in the microbial cell which are essential to its growth and survival $(13,138)$. Chitosan that enters the microbial cytosol may also interfere with mRNA synthesis by binding to the rDNA, and inhibiting protein synthesis (139). 
The therapeutic potential of CS rests with its ability to control bacterial growth, inhibit viral multiplication, and destroy fungi. This was highlighted in a publication by Muzzarelli et al. (140) who reported an inhibitory, bactericidal, and fungicidal activity by $\mathrm{N}$-carboxybutyl chitosan against 298 cultures of various pathogens. CS has demonstrated several advantages over other types of disinfectants such as higher antibacterial activity, a broader spectrum of activity, a higher killing rate, and a lower toxicity rate for mammalian cells $(13,141)$. Chitosan is insoluble in water and alkaline medium, and when neutralized with acids, can form water-soluble CS salts (13).

\subsubsection{Nanoparticles in Endodontics}

Nanoparticles have a diameter of $100 \mathrm{~nm}$ or less, and therefore exhibit a significantly greater surface area and charge density than powders. This offers them an advantage in antimicrobial therapy since it provides a significantly greater degree of interaction between the positively charged nanoparticles and the negatively charged bacterial cell surface. Nanoparticles have the ability to penetrate and extend their action into dentin microporosities, and areas of the root canal that are typically inaccessible to the commonly used endodontic irrigants (11).

Nanoparticles, such as $\mathrm{TiO} 2, \mathrm{ZnO}$, and fullerenes and their derivatives, have demonstrated a potential to generate singlet oxygen (11) and Klepac-Ceraj et al. (111) and Pagonis et al. (112) have shown a greater killing capacity of E. faecalis in planktonic 
and biofilm form by methylene blue loaded with poly(lactic-co-glycolic (PLGA) nanoparticles $(111,112)$. The future use of nanoparticles appears bright because they can be conjugated with other agents to further enhance their spectrum of use. Heavy metal nanoparticles such as copper oxide $(\mathrm{CuO})$, silver $(\mathrm{Ag})$ and $\mathrm{ZnO}$ have also been synthesized $(142,143)$. The copper ions released by $\mathrm{CuO}$ induce oxidative stress and membrane and DNA damage (144), while increased amount of zinc ions inhibit essential bacterial enzymes such as dehydrogenase, thereby impeding metabolic activity (145, 146). Silver ions inactivate protein and prevent DNA replication $(147,148)$. The disadvantage of using metal-based nanoparticles for root canal disinfection is its potential to cause tooth discolouration.

Antimicrobial properties for magnesium oxide $(\mathrm{MgO})$, calcium oxide $(\mathrm{CaO})$, and zinc oxide $(\mathrm{ZnO})$ powders have been cited and attributed to their ability to generate reactive oxygen species, such as hydrogen peroxide and superoxide anion radicals (11). Slurries of $\mathrm{MgO}$ and $\mathrm{CaO}$ have been reported to demonstrate a bactericidal effect against both Gram-positive and Gram-negative species (149) and a slurry of $\mathrm{ZnO}$ has been shown to have strong bacteriostatic properties against Gram-positive than Gram-negative bacteria (150). 


\subsubsection{Chitosan Nanoparticles in Endodontics}

Due to its broad antimicrobial spectrum and its low potential for inducing microbial resistance, the therapeutic use of CS has been the subject of many reports published in the scientific literature. Nanoparticle preparations have exhibited higher antibacterial activity than standard preparations (149). Chitosan nanoparticles (CSnp) are able to reduce biofilm bacteria, disrupt biofilm structure, and retain the antibacterial property after aging. CSnp also have displayed an increased polycationic/polyanionic interactions with the bacterial cell wall that leads to an increase in antibacterial activity (14). In a study by Shrestha et al. (151), efficacy of $\mathrm{CS}$ and $\mathrm{ZnO}$ nanoparticles against $E$. faecalis bacteria grown in planktonic and biofilm phases was evaluated. The rate of bacterial killing by CSnp was shown to be related to their concentration and their time of action. In this study bacteria in planktonic form were completely eliminated, but bacteria in biofilm managed to survive even after 72 hours of exposure. Interestingly, the nanoparticles tested retained their antibacterial properties for 92 hours.

Nanoparticles can also be incorporated into root canal sealers to improve their antimicrobial properties. Kishen et al. (14) investigated the antimicrobial properties of zinc-oxide eugenol ( $\mathrm{ZnOE}$ ) and resin-based sealers loaded with $\mathrm{CS}$ and $\mathrm{ZnO}$ nanoparticles. Findings indicated an improvement in the sealers' direct antibacterial property and increased ability of the antibacterial components of the sealers to leach out with the addition of nanoparticles. Furthermore, the addition of nanoparticles did not alter the flow characteristics of the sealer. 


\subsubsection{Chitosan Incorporation into Dentin}

Dentin can also be reinforced and stabilized with the incorporation of CS biopolymer into the collagen component of its matrix. When incorporated into collagen, CS increases the collagen resistance to enzymatic degradation $(12,15,16)$. Demineralization of dentin subsequent to the use of some intracanal irrigants, such as EDTA, or mild acids that are used for the removal of smear layer, exposes collagen on the surface of the root canal wall (152). Exposed collagen acts as an excellent substrate for adherence by many bacteria, including E. faecalis, and adherence is the first step involved in the formation biofilm. Exposed collagen is also substrate required for the survival of collagenase producing bacteria, many of which have been identified as putative endopathogens. Degradation of collagen, as mentioned previously, has the potential to change the physical properties of the dentin and predispose the tooth to fracture. Interaction of positively charged CS with negatively charged root canal dentin undermines adherence of the bacterial cells to the dentin and thus inhibits colonization and biofilm formation (14, 153). CS also enhances the cross linkage of collagen fibrils and therefore increases its resistance to enzymatic degradation. The stabilization of collagen by PDT and conjugated CS nanoparticles has been the subject of a number of publications.

Collagen biodegradation has been reported to slow down by incorporation of chitosan (154). Chitosan is a polysaccharide with similar structural characteristics to glycosaminoglycans, a major constituent of the extracellular matrix, making it an attractive candidate in tissue engineering. It is biocompatible, biodegradable, and 
bioactive. This polysaccharide has been shown to accelerate cell proliferation, promoting soft and hard tissue regeneration in wound-healing applications (154). Chitosan applied to collagen membrane, results in the formation of a thin membrane across the fibers. This membrane can protect the collagen from collagenase degradation (155). The interaction between chitosan and collagen has been found to be electrostatic, occurring between the carboxylate groups of collagen $\left(-\mathrm{COO}^{-}\right)$and the ammonium $\left(-\mathrm{NH}_{3}{ }^{+}\right)$functions of chitosan or by hydrogen bonding depending on the conditions (156). 


\title{
CHAPTER 3
}

3. Article

NOTE: The following article has been submitted for publication.

\section{Bioactive Chitosan Nanoparticles and Photodynamic Therapy}

\section{Inhibit Collagen Degradation in vitro}

\author{
Anousheh Persadmehr*, BSc, DDS, Calvin D. Torneck, DDS, MS, FRCD(C), \\ Dennis G. Cvitkovitch, BSc, PhD, Vanessa Pinto $\dagger, \mathrm{MSc}$, Ilana Talior†, PhD, \\ Mwayi Kazembe†, BSc, Suja Shrestha PhD, Christopher A. McCulloch†े, BSc, DDS, \\ FRCD(C), PhD, Anil Kishen, BDS, MDS, PhD \\ Faculty of Dentistry, University of Toronto, Toronto, ON \\ $\dagger$ Matrix Dynamics Group, University of Toronto, Toronto, ON
}

Correspondence: Dr. A. Kishen, Department of Endodontics, Faculty of Dentistry, University of Toronto, 124 Edward St., Toronto, Ontario M5G 1G6, Canada. E-mail address: anil.kishen@utoronto.ca. 
Key Words: dentin, chitosan, photodynamic therapy, collagenase, nanoparticles

Acknowledgements: Supported by AAE Foundation, CAE Endowment Fund, Alpha Omega Foundation of Canada, CIHR operating grant to CAM (MOP-418228) and Canada Research Chair (CAM). The authors thank the Kishen Lab and the Matrix Dynamics Group for their valuable assistance in the conduct of these experiments. The authors deny any conflicts of interest related to this study. 


\begin{abstract}
Introduction: Collagen is the major structural protein of human dentin. Degradation of collagen by bacterial enzymes can facilitate microbial penetration, compromise structural/interfacial integrity and lower resistance to fracture of dentin. We evaluated the ability of photodynamic therapy (PDT), bioactive chitosan nanoparticles (CSnp), or PDT in combination with CSnp, to inhibit bacterial collagenase-mediated degradation of collagen.
\end{abstract}

Methods: Rat type 1 fibrillar collagen matrices were untreated or treated with $2.5 \%$ glutaraldehyde (GD), 2.5\% GD followed by 1\% CSnp, 1\% CSnp, PDT (Rose Bengal activated with $540 \mathrm{~nm}$ light at $40 \mathrm{~J} / \mathrm{cm}^{2}$ ), or $1 \%$ CSnp followed by PDT. Samples, except those used as untreated controls, were exposed to Clostridium histolyticum collagenase (125 CDU/ml) for $24 \mathrm{~h}$. The soluble digestion products were assessed by hydroxyproline assay and the remaining adherent collagen was quantified by picrosirius red (PSR) staining. Fourier Transform Infrared Spectroscopy (FTIR), immunoblotting and Scanning Electron Microscopy were used to study the interaction between CSnp/PDT with type 1 collagen. The data were analyzed by one-way ANOVA.

Results: As assessed by hydroxyproline release into the medium, collagen treated with CSnp, PDT, or a combination of CSnp and PDT, exhibited less degradation than untreated controls $(3.6,1.7$, and 7.9 fold reduction, respectively; $p<0.05)$. Compared with all other treatments, GD-treated collagen was the most resistant to collagenolytic degradation (239.6-fold reduction; $p<0.05)$. The abundance of post-treatment residual collagen, as measured by PSR staining, was inversely related to the extent of collagen degradation. Analysis of collagen cross-links with FTIR showed that PDT or GD 
treatments enhanced collagen cross-linking. Immunoblotting of sedimented CSnp indicated that CSnp and collagenase bound with low affinity. However, CSnp-bound collagenase showed a significant reduction in collagenolytic activity compared with controls $(p<0.05)$.

Conclusion: Photochemical cross-linking by PDT, and binding to CSnp will inhibit the collagenolytic activity of collagenase on type 1 collagen. In combination, PDT and CSnp treatment of root dentin may inhibit collagenolytic degradation of exposed dentin collagen. 


\section{Introduction}

Primary apical periodontitis is a manifestation of pathological processes induced by the interaction of host tissues with microorganisms and their metabolites in infected root canals (1). Effective control of pathogenic microorganisms and mechanical stabilization of root canal dentin can significantly improve the long-term survival of endodonticallytreated teeth (2-4). Conventional treatments typically employ a chemo-mechanical approach, which involves a combination of root canal debridement, shaping of the root canal with metallic instruments, irrigation and/or dressings with chemical disinfectants, and chemical preparation of the dentin wall to optimize sealing of the root canal system with a cement and core filling. Current protocols that use this approach are not completely successful in eliminating pathogenic bacterial biofilms from the root canal system (5) nor do they address the damage to dentin caused by bacterial proteases $(6,7)$ and chemicals that are used in most of those treatment protocols $(8,9)$. Indeed, disease and treatment-mediated damage to dentin can result in interfacial failures at the filling material/dentin interface and diminish mechanical strength of the dentin, which increases the risk of fracture $(3,10)$. All of these alterations to dentin may compromise the longterm survival of endodontically-treated teeth. The limitations of these treatment approaches highlight the need for new strategies for clinical management of teeth with endodontic infections that can not only disinfect the root canals but also preserve and possibly enhance the chemical/mechanical stability of dentin. One promising method of achieving these goals is the use of photodynamic therapy (PDT) in combination with polycationic chitosan nanoparticles (CSnp). 
Photodynamic therapy employs a specific wavelength of light to activate a nontoxic dye (photosensitizer), leading to the formation of reactive oxygen species. These reactive oxygen molecules can damage bacterial proteins, membrane lipids and nucleic acids, which promote bacterial cell death (11). Further, PDT may enhance cross-linking of collagen fibrils in the dentin matrix and thereby improve dentin stability $(12,13)$. The effectiveness of PDT in root canal disinfection has been demonstrated by in vitro and in vivo studies (14-16). In addition, PDT-generated reactive oxygen species increase the number of intermolecular cross-links between adjacent collagen fibrils, which may enhance the resistance of collagen to enzymatic degradation at diseased or chemically altered sites. Since collagen is the principal structural protein of the dentin matrix, increased collagen cross-linking could improve the mechanical properties of dentin affected by endodontic infection $(12,17)$.

Dentin can be reinforced by the incorporation of biopolymers into the collagenassociated fraction of the matrix. Chitosan (CS) (poly $(1,4)$,-b-D-glucopyranosamine) is a natural hydrophilic polycationic polymer, derived from alkaline deacetylation of chitin present in the shells of crustaceans (18). Chitosan is markedly biocompatible, exhibits a broad antimicrobial spectrum $(18,19)$ and may increase the resistance of collagen to enzymatic degradation $(12,20,21)$. The use of PDT in combination with chitosan nanoparticles (CSnp) may provide an effective approach to overcoming two of the major shortcomings of currently used protocols in endodontic treatments namely, their limited effectiveness against root canal biofilms and their lack of stabilization of root canal dentin. Currently the mechanisms by which PDT and CSnp affect the stability of dentin 
collagen are not defined. Our purpose was to examine how PDT and CSnp affect collagen degradation in vitro by bacterial collagenase. 


\section{Material and Methods}

Preparation and Treatment of Collagen Gels

Type 1 rat tail collagen (BD Biosciences, Bedford MA) was polymerized into gels according to the manufacturer's instructions. Briefly, collagen in $0.02 \mathrm{~N}$ acetic acid was neutralized with $1 \mathrm{~N} \mathrm{NaOH}$; 10x PBS was added to obtain a final collagen concentration of $3 \mathrm{mg} / \mathrm{ml}$. An aliquot (200 ul) of this solution was placed in each well of a 24-well micro-plate and allowed to gel at $37^{\circ} \mathrm{C}$ for $30 \mathrm{~min}$. The CSnp were synthesized using an ionic gelation method and their physical characteristics were standardized as described previously (19). In short, CS was dissolved in $1 \mathrm{v} / \mathrm{v} \%$ HOAc solution at a concentration of $0.5 \mathrm{w} / \mathrm{v} \%$, and the $\mathrm{pH}$ was raised to 4.6 to 5 with $10 \mathrm{~N} \mathrm{NaOH}$. CSnp was formed upon adding $5 \mathrm{~mL} 0.25 \%$ sodium tripolyphosphate in water to $15 \mathrm{~mL}$ CS solution under stirring at a speed of $1,000 \mathrm{rpm}$. The nanoparticles were separated by centrifugation at 20,000 rpm for 30 minutes. The supernatant was discarded, and the CSnp was extensively rinsed with water to remove any $\mathrm{NaOH}$ and then freeze-dried before further use.

After conduct of preliminary experiments examining the time and concentration necessary to optimize collagen degradation by collagenase, gels were divided into the following groups: Group A - native gel (untreated negative control); B - native gel (collagenase-treated control); Group C - treatment with $1 \mathrm{~mL}$ 2.5\% glutaraldehyde (GD) for $6 \mathrm{~h}$; Group D - treatment with $1 \mathrm{ml} 2.5 \% \mathrm{GD}$, washed with de-ionized water after $6 \mathrm{~h}$ and treated with $1 \mathrm{~mL} \mathrm{1 \%}$ chitosan nanoparticles (CSnp) for $15 \mathrm{~min}$; Group E- treatment with $1 \mathrm{~mL} \mathrm{1 \%} \mathrm{CSnp} \mathrm{for} 15 \mathrm{~min}$; Group F- treatment by PDT that required 15 min incubation at room temperature in $1 \mathrm{~mL}$ of $10 \mathrm{uM}$ Rose Bengal (RB) (Sigma-Aldrich, St. Louis, MO), removal of excess RB, then exposure to non-coherent light at $540 \mathrm{~nm}$ for 14 
$\min \left(40 \mathrm{~J} / \mathrm{cm}^{2}\right)$ (LumaCare, Newport Beach, CA); Group G- treatment with $1 \mathrm{~mL} \mathrm{1 \%}$

CSnp for $15 \mathrm{~min}$, washed with $1 \mathrm{~mL}$ de-ionized water, followed by PDT.

After each treatment, gels (with the exception of Group A) were washed twice with $1 \mathrm{~mL}$ of de-ionized water to remove excess reagent, then exposed to $20 \mathrm{uL}$ of $125 \mathrm{CDU} / \mathrm{mL}$ Clostridium histolyticum collagenase (Sigma-Aldrich, St. Louis, MO) for $24 \mathrm{~h}$. Gels in Group A collagen gels were treated with $20 \mathrm{uL}$ PBS for $24 \mathrm{~h}$. Gels in Group C and D served as chemically cross-linked collagen controls. The supernatants containing collagen degradation products released by collagenase degradation were collected in $200 \mathrm{uL}$ PBS and stored at $-80^{\circ} \mathrm{C}$ later for future study.

\section{Hydroxyproline Assay and Picrosirius Red Staining}

After collagenase degradation of collagen, the amount of hydroxyproline (OH-Pro) released was quantified as described by Bidlingmeyer et al. (22) with minor modifications at the Advanced Protein Technology Center (APTC) of The Hospital for Sick Children. Briefly, free amino acid analysis was performed on a Waters Acquity HPLC system. Sample aliquots were transferred into a glass culture tube and dried under vacuum. The dried sample was treated with a methanol:water:triethylamine (2:2:1) redrying solution that was then vortex-mixed and dried under vacuum for $15 \mathrm{~min}$. The sample was derivatized for $20 \mathrm{~min}$ at room temperature with a methanol: water: triethylamine: phenylisothiocyanate (PITC) (7:1:1:1) derivatizing solution. The derivatizing solution was removed and placed under vacuum for $15 \mathrm{~min}$. The sample was washed with re-drying solution, vortex-mixed and dried under vacuum for $15 \mathrm{~min}$. The derivatized sample was dissolved in sample diluent (pH 7.4) and an aliquot was injected into the column, running on a modified PICO-TAG gradient at $48^{\circ} \mathrm{C}$. The derivatized 
amino acids were detected at $254 \mathrm{~nm}$. Data were collected, stored and processed using Waters Empower 2 Chromatography software.

The gels from which medium was collected for hydroxyproline assay, were washed and stained with $0.1 \%$ Sirius red in saturated picric acid (Electron Microscopy Sciences, Hatfield, PA) for $2 \mathrm{~h}$ at room temperature to assess the amount of residual collagen. Excess dye in each well was removed with two, $0.05 \%$ acetic acid washes and the picrosirius dye that adsorbed to the collagen was dissolved in $1 \mathrm{ml}$ of $2 \%$ sodium dodecyl sulfate solution and incubated overnight at $37^{\circ} \mathrm{C}$. The amount of dye obtained from each gel was quantified with a spectrophotometer (Epoch Micro-Volume Spectrophotometer System, Winooski, VT) at $555 \mathrm{~nm}$ using Gen5 data analysis software.

\section{Analysis of Collagen Cross-Linking}

Gels in GD, PDT, and untreated collagen groups were vacuum-desiccated, treated with liquid nitrogen and ground into small particles. The ground specimens were mixed with potassium bromide $(1: 100 \mathrm{w} / \mathrm{w})$ and subjected to Fourier transform infrared (FTIR) spectroscopy (16 $\mathrm{cm}^{-1}$ resolution, 100 scans per sample) (Shimadzu, Kyoto, Japan) to assess collagen cross-links.

\section{Immunoblotting}

Associations between CSnp and collagenase were assessed by first incubating CSnp with collagenase solutions. The solutions were centrifuged and the sediments and supernatants were immunoblotted for collagenase. Briefly, an aliquot (1 $\mathrm{ml}$ of $1 \% \mathrm{CSnp}$ ) was incubated with $200 \mu \mathrm{l}$ of $125 \mathrm{CDU} / \mathrm{ml}$ collagenase for $1 \mathrm{~h}$ at $37^{\circ} \mathrm{C}$ and then sedimented $(30,000 \mathrm{~g}$ for $1 \mathrm{~h}$ at $\left.15^{\circ} \mathrm{C}\right)$. CSnp pellets were re-suspended in $1 \mathrm{ml}$ of de-ionized water. Supernatants were serially diluted at concentrations of 1:2,1:4, 1:8 and 1:16. Aliquots (400 $\mu 1)$ of each 
of the supernatant and pellet-associated samples were immunoblotted for collagenase. The remaining volume in each sample was applied to the surface of prepared collagen gels for 24 hours at $37^{\circ} \mathrm{C}$. Digestion products were removed and assessed with the hydroxyproline assay.

Scanning Electron Microscopy (SEM) Evaluation

Gels were analyzed by scanning electron microscopy (SEM) (Hitachi S-2500, Sapporo, Japan) at $10 \mathrm{kV}$. Gels were washed with PBS and fixed in 4\% GD in sodium cacodylate buffer at $4^{\circ} \mathrm{C}$ for $3 \mathrm{~h}$ then serially dehydrated with increasing concentrations of ethanol, critical point dried and sputter-coated with gold. These specimens were used to subjectively assess the ultrastructure of collagen gel surfaces.

\section{Data Analysis}

All experiments were performed in triplicate. Means and standard errors of the mean of continuous variables were calculated. Descriptive data and statistical analyses were performed using SPSS (version 16.0, Chicago, IL). The difference between the control group and the treatment groups was analyzed by one-way ANOVA. Statistical significance was set at $p<0.05$. 


\section{Results}

\section{Degradation of Collagen Gels}

The highest amount of hydroxyproline release into the medium was detected in native collagen gels treated with collagenase (Fig. 1A). PSR assays of the collagen remaining in these gels showed no detectable staining (Fig. 1B). Collagen gels treated with PBS alone or gels that were pre-treated with $2.5 \%$ GD prior to collagenase treatment, exhibited no detectable release of hydroxyproline into the medium. These same samples also exhibited the highest amount of remaining collagen in the gel as detected by PSR staining. Collagen gels treated with CSnp alone or with PDT alone exhibited $<30 \%$ and $<60 \%$ of hydroxyproline release compared with native collagen gels treated with collagenase, respectively $(p<0.05)$. Gels treated with CSnp and PDT displayed $<15 \%$ of hydroxyproline release compared with native collagen gels treated with collagenase $(p<0.05)$. While the amount of residual collagen was greater with the combined CSnp/PDT treatment than with single CSnp- or PDT-only treatments, these differences were not significantly different $(p>0.2)$.

\section{Collagen Cross-Linking}

The amide I $\left(\sim 1656 \mathrm{~cm}^{-1}\right)$ and amide II $\left(\sim 1550 \mathrm{~cm}^{-1}\right)$ bands of FTIR spectra were analyzed to assess cross-linking in PDT-treated gels $(20,23)$. FTIR spectra showed reduced amide I peak relative to the amide II peak in GD- and PDT-treated gels, when compared with untreated collagen gels. This spectral change indicated increased crosslinking of collagen gels treated with GD or PDT (Fig. 2). 


\section{Collagenase Association with Chitosan}

We determined whether the inhibition of collagenase activity by CSnp was attributable to association of collagenase with CSnp and presumptive blockade of catalytic activity. We incubated an aliquot of $1 \% \mathrm{CSnp}$ with $200 \mu \mathrm{l}$ of $125 \mathrm{CDU} / \mathrm{mL}$ collagenase for $1 \mathrm{~h}$ at $37^{\circ} \mathrm{C}$, sedimented the nanoparticles and retained the supernatant. Serial dilutions of the supernatant and the material eluted from the nanoparticles were incubated with collagen gels for one day and hydroxyproline release was measured. These data showed that the collagenase activity associated with the nanoparticles was only a small fraction of the collagenase activity present in the supernatants for all dilutions (Fig. 3A). These same samples were immunoblotted for collagenase, which showed multiple, autocatalytic cleavage fragments of bacterial collagenase (MW=68-130 kDa) in all samples (Fig. 3B). The relative amount of collagenase eluted from the chitosan nanoparticles was comparable to the amount of collagenase detected in the 1:16 dilution of the supernatant (Fig. 3B). Collagenase activity, as measured by the hydroxyproline assay, indicated that the degradation activity of the collagenase associated with the nanoparticles was $\sim 35 \%$ of that detected in a 1:16 dilution of the supernatant sample $(p<0.05)$ (Fig. 3A).

\section{Scanning Electron Microscopy}

Scanning electron microscopy analysis of the collagen gels after collagenase treatments showed infiltration of the collagen network by CSnp (Fig. 4D). In the GD and PDTtreated samples, there was ultrastructural evidence of enhanced inter- and intra-fibril cross-linking, as suggested by the physical association of adjacent fibrils (Fig. 4C, E). In gels treated with CSnp and PDT, the CSnp were also observed to be closely associated with collagen fibrils (Fig. 4F). 


\section{Discussion}

We examined here fundamental aspects of a treatment approach that may limit collagen degradation, stabilize the dentin matrix and conserve the physical properties of dentin (12). Our principal finding is that chitosan nanoparticles and photodynamic therapy inhibit the activity of bacterial collagenases in vitro. The collagenase inhibitory property of chitosan nanoparticles and the ability of photodynamic therapy to cross-link collagen fibrils when infiltrated by chitosan nanoparticles, rationalize this combined approach for improving the structural integrity of demineralized root canal dentin.

In mammals, type 1 collagen is the principal structural protein $(\sim 90 \%)$ of the dentin organic matrix (24) and contributes to the viscoelasticity, ultrastructural stability and tensile strength of dentin $(3,25)$. The integrity of collagen fibrils is dependent in part on the formation of intermolecular cross-links between collagen molecules (26), which increase the resistance of collagen to proteolytic digestion. Collagenases are endopeptidases produced by mammalian and bacterial cells that cleave native, triple helical collagen (27). Bacterial collagenases, unlike human collagenases, exhibit limited substrate specificity, degrade most types of collagen types and can efficiently cleave cross-linked collagen and the triple helical regions of collagen molecules. These cleavages lead to degradation of collagen fibrils and loss of the structural strength of the dentin matrix. Accordingly, inhibition of collagenases expressed by bacterial biofilms in infected dentin is a rational approach for improving treatment outcomes in endodontic therapy. 
In evaluating the ability of chitosan nanoparticles and photodynamic therapy to inhibit collagen degradation in vitro, we measured soluble hydroxyproline to quantify the extent of collagenase-mediated degradation in collagen gels (28). Hydroxyproline is an abundant imino acid of collagen that is released during collagen degradation and provides a highly sensitive and reproducible measure to compare the various treatments for inhibition of collagenase activity. The ability of the CSnp and PDT to inhibit collagenase activity was also assessed by spectrophotometric measurement of picrosirius red, a dye that binds tightly to intact collagen fibrils. While our preliminary experiments indicated that assessment of residual collagen by picrosirius red staining after collagenase treatments was not as sensitive an assay as hydroxyproline release, the combined analysis of picrosirius red staining in the collagen remaining attached to the microwell plate and hydroxyproline release into the medium, enhanced our insight into the kinetics and mechanisms by which chitosan nanoparticles and photodynamic therapy inhibit collagen degradation. For example, in the collagen gels prepared for our test study subjected to collagenase degradation for more than one day, there was progressive, time-dependent release of hydroxyproline into the medium, which was not detected with the picrosirius red staining of residual collagen in the gels, presumably because of the loss of fibrillar collagen that occurred within the first day of collagenase treatment.

C. histolyticum collagenase is an efficient and rapidly acting collagen-degrading protease and was used in our study as a prototypical collagenase produced by microorganisms frequently found in an infected root canal $(29,30)$. Preliminary trials showed that $C$. histolyticum collagenase generated very high levels of hydroxyproline release and virtually undetectable levels of picrosirius red staining in the residual 
collagen after $24 \mathrm{~h}$ incubations. These properties rationalized the use of C. histolyticum collagenase as our test enzyme to examine the potential inhibition of collagen degradation by chitosan nanoparticles and photodynamic therapy. Notably, for each of the treatment protocols, there was evidence of enhanced resistance of collagen to enzymatic degradation although there was no statistically significant difference between the treatments.

We used glutaraldehyde, which cross-links lysine residues in adjacent proteins and inhibits collagenase activity, as a control for comparing inhibition of collagen degradation. Specifically, the aldehyde residues of glutaraldehyde react with the amine groups of lysine or hydroxylysine residues in collagen to produce a network of crosslinks $(31,32)$ that increases the resistance of collagen to enzymatic degradation $(31,33)$. While not practical for clinical use because of the length of exposure time $(\sim 6 \mathrm{~h})$ and its inherent toxicity (34), glutaraldehyde was a useful control in our experimental design as it inhibited collagenase degradation by $\sim 240$-fold $(p<0.05)$.

Reactive oxygen species generated by photodynamic therapy can interact with amino and imino acids in collagen that are involved in intermolecular cross-links (12). The FTIR analysis showed a reduced amide I peak relative to the amide II peak, which indicates conversion of a free $\mathrm{NH}_{2}$ group to $-\mathrm{NH}$ group $(20,23)$ and suggests the formation of additional cross-links between adjacent collagen fibrils. Accordingly, the use of photodynamic therapy in the treatment approach examined here may contribute to collagenase inhibition by virtue of its expansion of the number of collagen cross-links. 
In collagen gels treated with CSnp alone, there was increased resistance to collagenase-mediated degradation of collagen, as reported earlier (35). CSnp exhibit electrostatic attraction to collagen that is dependent on local environmental conditions, including the relative proximity of the carboxyl groups of collagen (- $\left.\mathrm{COO}^{-}\right)$to the amino $\left(-\mathrm{NH}_{3}{ }^{+}\right)$groups of chitosan and by chitosan associations mediated by hydrogen bonding (35). The spatial co-localization between CSnp and collagen fibrils and stabilized collagen ultrastructure was noted in the scanning electron microscopy. The immunoblotting analyses indicated low affinity associations between chitosan nanoparticles and collagenase, which appears sufficient to reduce collagenase activity by $\sim 65 \%$ (2.8-fold reduction), as detected in hydroxyproline release assays.

Each of the three experimental protocols PDT, CSnp, and a combination of PDT and CSnp examined in this study, appears to be more effective in protecting dentin against collagenase than current endodontic treatment protocols, which employ acids, chelating agents, sodium hypochlorite, and calcium hydroxide $(8,9)$. These previous studies show that these agents not only fail to preserve the structural strength and mechanical properties of dentin but also tend to undermine dentin. Additionally, agents that expose collagen in the dentin matrix also increase the susceptibility of the matrix to enzymatic degradation by bacterial collagenases. Based on our current findings and the potential of photodynamic therapy combined with chitosan nanoparticles to reduce the microbial load in infected root canals $(12,14,15)$, further investigation is warranted towards clinical use. 


\section{Conclusions}

The ability of CSnp and PDT to inhibit the collagenolytic activity of collagenases could improve the long-term structural integrity of root canal dentin. 


\section{References}

1. Nair PN. Apical periodontitis: A dynamic encounter between root canal infection and host response. Periodontol 2000 1997;13:121-48.

2. Haapasalo M, Shen Y. Current therapeutic options for endodontic biofilms. Endodontic Topics 2010;22(1):79-98.

3. Kishen A. Mechanisms and risk factors for fracture predilection in endodontically treated teeth. Endodontic Topics 2006;13(1):57-83.

4. Kishen A. Advanced therapeutic options for endodontic biofilms. Endodontic Topics 2010;22(1):99-123.

5. Nair PN. On the causes of persistent apical periodontitis: A review. Int Endod J 2006;39(4):249-81.

6. Tang $\mathrm{W}, \mathrm{Wu} \mathrm{Y}$, Smales RJ. Identifying and reducing risks for potential fractures in endodontically treated teeth. J Endod. 2010;36(4):609-17.

7. Ferrari M, Mason PN, Goracci C, Pashley DH, Tay FR. Collagen degradation in endodontically treated teeth after clinical function. J Dent Res 2004 May;83(5):414-9.

8. Saleh AA, Ettman WM. Effect of endodontic irrigation solutions on microhardness of root canal dentine. J Dent 1999;27(1):43-6.

9. Qian W, Shen Y, Haapasalo M. Quantitative analysis of the effect of irrigant solution sequences on dentin erosion. J Endod 2011;37(10):1437-41.

10. Niu W, Yoshioka T, Kobayashi C, Suda H. A scanning electron microscopic study of dentinal erosion by final irrigation with EDTA and $\mathrm{NaOCl}$ solutions. Int Endod J 2002;35(11):934-9.

11. Hamblin MR, Hasan T. Photodynamic therapy: A new antimicrobial approach to infectious disease? Photochemical \& photobiological sciences: Official journal of the European Photochemistry Association and the European Society for Photobiology 2004;3(5):436-50.

12. Shrestha A, Friedman S, Kishen A. Photodynamically crosslinked and chitosan-incorporated dentin collagen. J Dent Res 2011;90(11):1346-51. 
13. Wollensak G, Iomdina E. Long-term biomechanical properties of rabbit cornea after photodynamic collagen crosslinking. Acta Ophthalmol 2009;87(1):48-51.

14. Soukos NS, Chen PS, Morris JT, Ruggiero K, Abernethy AD, Som S, et al. Photodynamic therapy for endodontic disinfection. J Endod 2006;32(10):979-84.

15. Williams JA, Pearson GJ, Colles MJ. Antibacterial action of photoactivated disinfection $\{P A D\}$ used on endodontic bacteria in planktonic suspension and in artificial and human root canals. J Dent 2006;34(6):363-71.

16. Garcez AS, Nunez SC, Hamblin MR, Ribeiro MS. Antimicrobial effects of photodynamic therapy on patients with necrotic pulps and periapical lesion. J Endod 2008;34(2):138-42.

17. Bedran-Russo A, Pereira PN, Duarte WR, Drummond JL, Yamauchi M. Application of crosslinkers to dentin collagen enhances the ultimate tensile strength. Journal of biomedical materials research. Part B, Applied biomaterials 2007;80(1):268-72.

18. Rabea EI, Badawy ME, Stevens CV, Smagghe G, Steurbaut W. Chitosan as antimicrobial agent: Applications and mode of action. Biomacromolecules 2003;4(6):1457-65.

19. Kishen A, Shi Z, Shrestha A, Neoh KG. An investigation on the antibacterial and antibiofilm efficacy of cationic nanoparticulates for root canal disinfection. J Endod 2008;34(12):1515-20.

20. Wang XH, Li DP, Wang WJ, Feng QL, Cui FZ, Xu YX, et al. Crosslinked collagen/chitosan matrix for artificial livers. Biomaterials 2003;24(19):3213-20.

21. Madhavan K, Belchenko D, Motta A, Tan W. Evaluation of composition and crosslinking effects on collagen-based composite constructs. Acta Biomater 2010;6(4):1413-22.

22. Bidlingmeyer BA, Cohen SA, Tarvin TL. Rapid analysis of amino acids using pre-column derivatization. Journal of chromatography 1984;336(1):93-104.

23. Rafat M, Li F, Fagerholm P, Lagali NS, Watsky MA, Munger R, et al. PEG-stabilized carbodiimide crosslinked collagen-chitosan hydrogels for corneal tissue engineering. Biomaterials 2008;29(29):3960-72.

24. Tjäderhane L, Carrilho MR, Breschi L, Tay FR, Pashley DH. Dentin basic structure and composition-an overview. Endodontic Topics 2012;20:3-29. 
25. Miguez PA, Pereira PN, Atsawasuwan P, Yamauchi M. Collagen cross-linking and ultimate tensile strength in dentin. J Dent Res 2004;83(10):807-10.

26. Rivera EM, Yamauchi M. Site comparisons of dentine collagen cross-links from extracted human teeth. Arch Oral Biol 1993;38(7):541-6.

27. Sottrup-Jensen L, Birkedal-Hansen H. Human fibroblast collagenase- $\alpha$-macroglobulin interactions. localization of cleavage sites in the bait regions of five mammalian $\alpha$ macroglobulins. J Biol Chem 1989;264(1):393-401.

28. Supuran CT, Scozzafava A, Clare BW. Bacterial protease inhibitors. Medicinal research reviews 2002;22(4):329-72.

29. Sundqvist G, Carlsson J, Hanstrom L. Collagenolytic activity of black-pigmented bacteroides species. J Period Res 1987;22(4):300-6.

30. Odell LJ, Baumgartner JC, Xia T, David LL. Survey for collagenase gene prtC in porphyromonas gingivalis and porphyromonas endodontalis isolated from endodontic infections. J Endod 1999;25(8):555-8.

31. Sung HW, Huang DM, Chang WH, Huang RN, Hsu JC. Evaluation of gelatin hydrogel crosslinked with various crosslinking agents as bioadhesives: In vitro study. J Biomed Mat Res 1999;46(4):520-30.

32. Ritter AV, Swift EJ, J., Yamauchi M. Effects of phosphoric acid and glutaraldehyde-HEMA on dentin collagen. European J Oral Sciences 2001;109(5):348-53.

33. Nimni ME. Bioprosthesis derived from crosslinked and chemically modified collagenous tissues. Nimni ME. 1988:1; 1,38; 38.

34. Han B, Jaurequi J, Tang BW, Nimni ME. Proanthocyanidin: A natural crosslinking reagent for stabilizing collagen matrices. J Biomed Mat Res Part A 2003;65(1):118-24.

35. Taravel MN, Domard A. Relation between the physicochemical characteristics of collagen and its interactions with chitosan: I. Biomat 1993;14(12):930-8. 


\section{Figure Legends}

Figure 1. A. Hydroxyproline Assay. Mean $( \pm \mathrm{SE})$ concentration of hydroxyproline $(\mathrm{OH}-$ Pro) released after collagen gel treatments and degradation with $C$. histolyticum for 24 hrs. Collagen gels were treated with glutaraldehyde (GD), chitosan nanoparticles (CSnp), photodynamic therapy (PDT) or CSnp + PDT. Significant difference $(p<0.05)$ noted between GD, CSnp, PDT, and CSnp + PDT treated gels and untreated controls as indicated by *. B. Picrosirius Red Stain. Amount of collagen gel remaining after bacterial digestion was quantified with a spectrophotometer (absorption-555 nm). There was significantly $(p<0.05)$ more collagen in undigested controls $(\mathrm{A})$ and after treatment with GD, CSnp, CSnp + PDT compared with samples without treatment. * indicates significant difference from designated control group.

Figure 2. FTIR spectra. In the photodynamic (PDT) and glutaraldehyde (GD)- treated collagen gels, the amide I $\left(\sim 1656 \mathrm{~cm}^{-1}\right)$ band was decreased relative to the amide II $\left(\sim 1550 \mathrm{~cm}^{-1}\right)$ band, in contrast to the untreated collagen sample, which indicated increased cross-linking. 
Figure 3. A. Hydroxyproline Assay. Mean concentration of $\mathrm{OH}-\mathrm{Pro}$ released was significantly $(p<0.05)$ less in the samples with chitosan nanoparticles $(\mathrm{CSnp})$-associated collagenase than all diluted supernatant collagenase. * indicates significant $(p<0.05)$ difference compared with all other diluted supernatant samples. B. Immunoblot of collagenase. The association between C. histolyticum collagenase and chitosan nanoparticles (CSnp) was assessed by co-incubation for $1 \mathrm{hr}$. After separation of the nanoparticles from the supernatant by sedimentation, the abundance of collagenase in serially-diluted supernatants and sedimented nanoparticles was determined by immunoblotting. The relative amount of collagenase associated with CSnp was a very small fraction of the collagenase remaining in the supernatant.

Figure 4. SEM images. A. Native collagen gel displaying prominent three-dimensional fibril network. B. Collagen gel exposed to C. histolyticum collagenase displaying significant reduction in fibril network. C. SEM of native gel treated with GD and digested with collagenase displaying intact fibril network with altered surface morphology in the form of globules (arrow). D. Collagen gel treated with CSnp with subsequent collagnenase degradation displaying intimate association of nps and collagen fibrils (arrow). E. Photodynamic-treated (PDT) collagen gels, displaying more abundant morphological evidence for cross-links between adjacent collagen fibrils (circle) following degradation with collagenase. F. SEM of CSnp + PDT treated collagen gel following digestion with collagenase displaying $\mathrm{np}$ aggregates in intimate association with collagen fibrils (arrow). 
Figure 1.
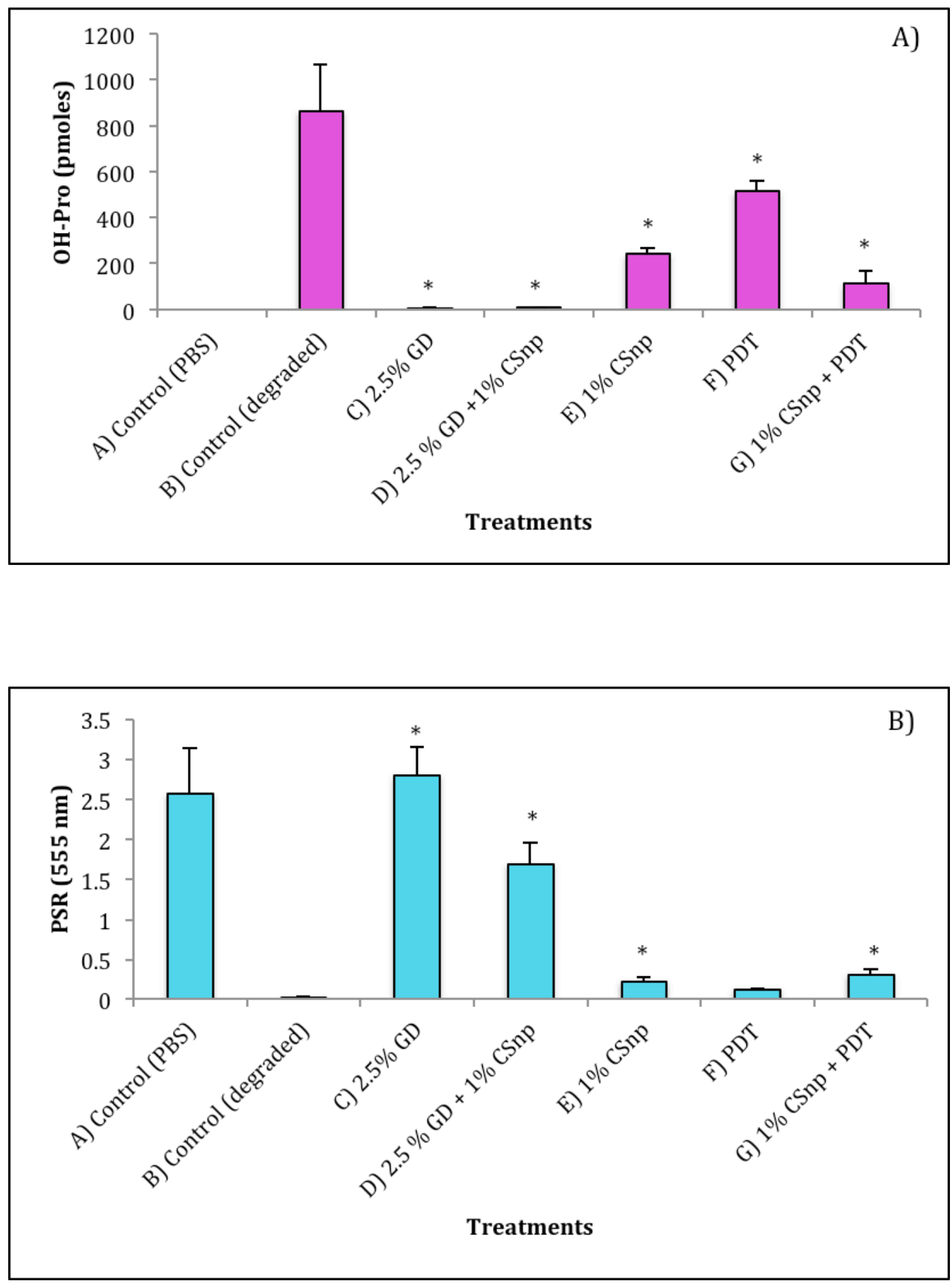
Figure 2.

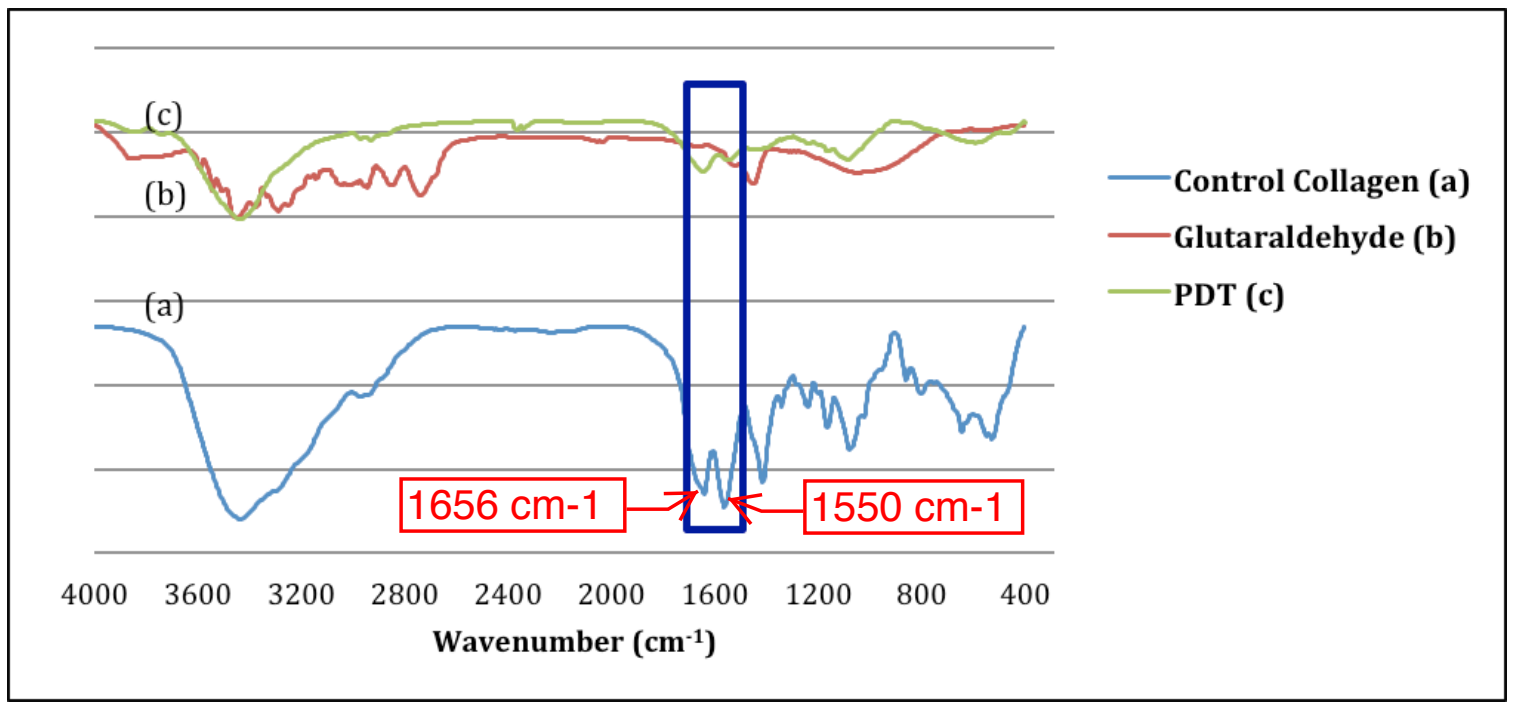


Figure 3.
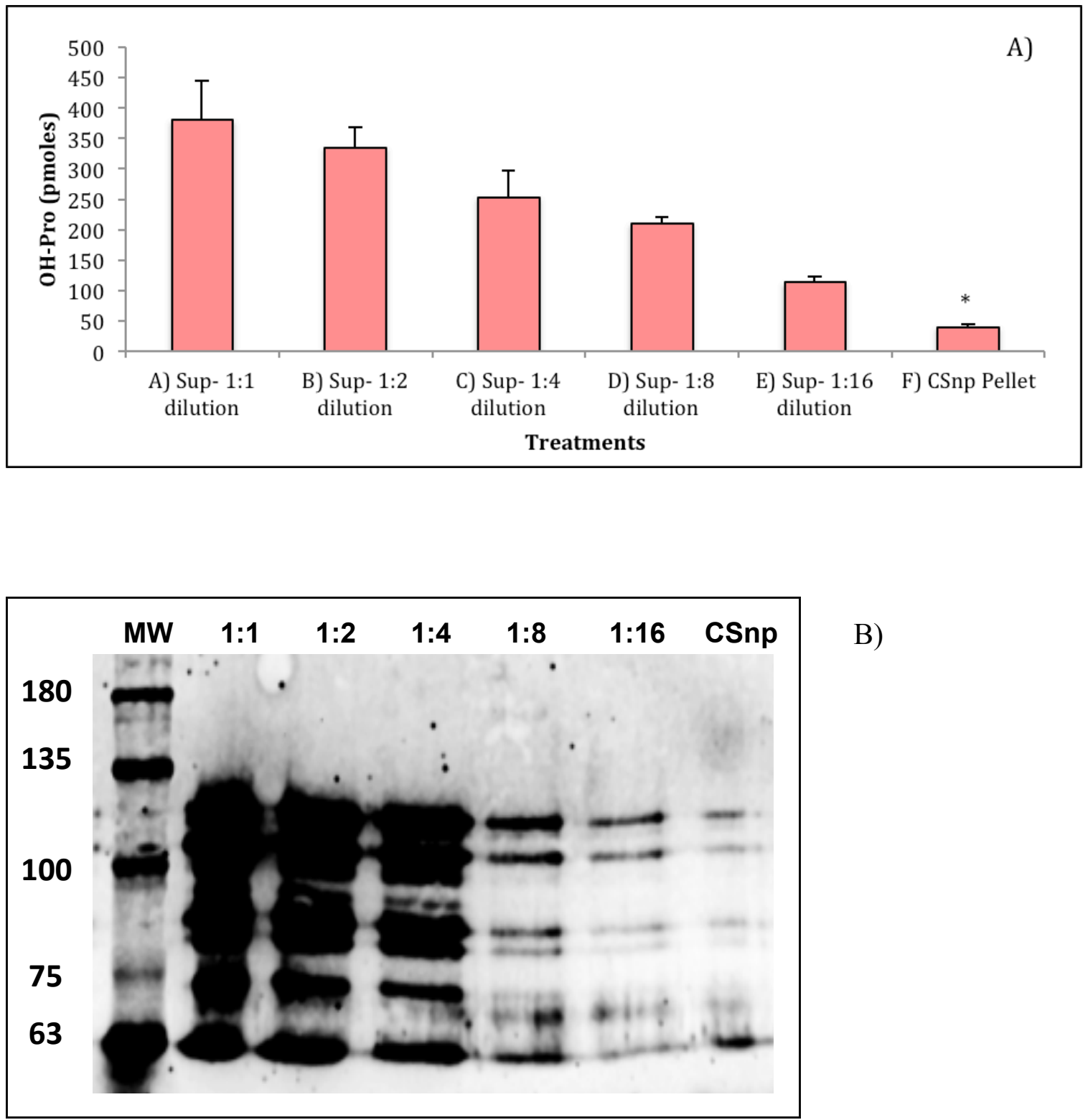

B) 


\section{Figure 4.}
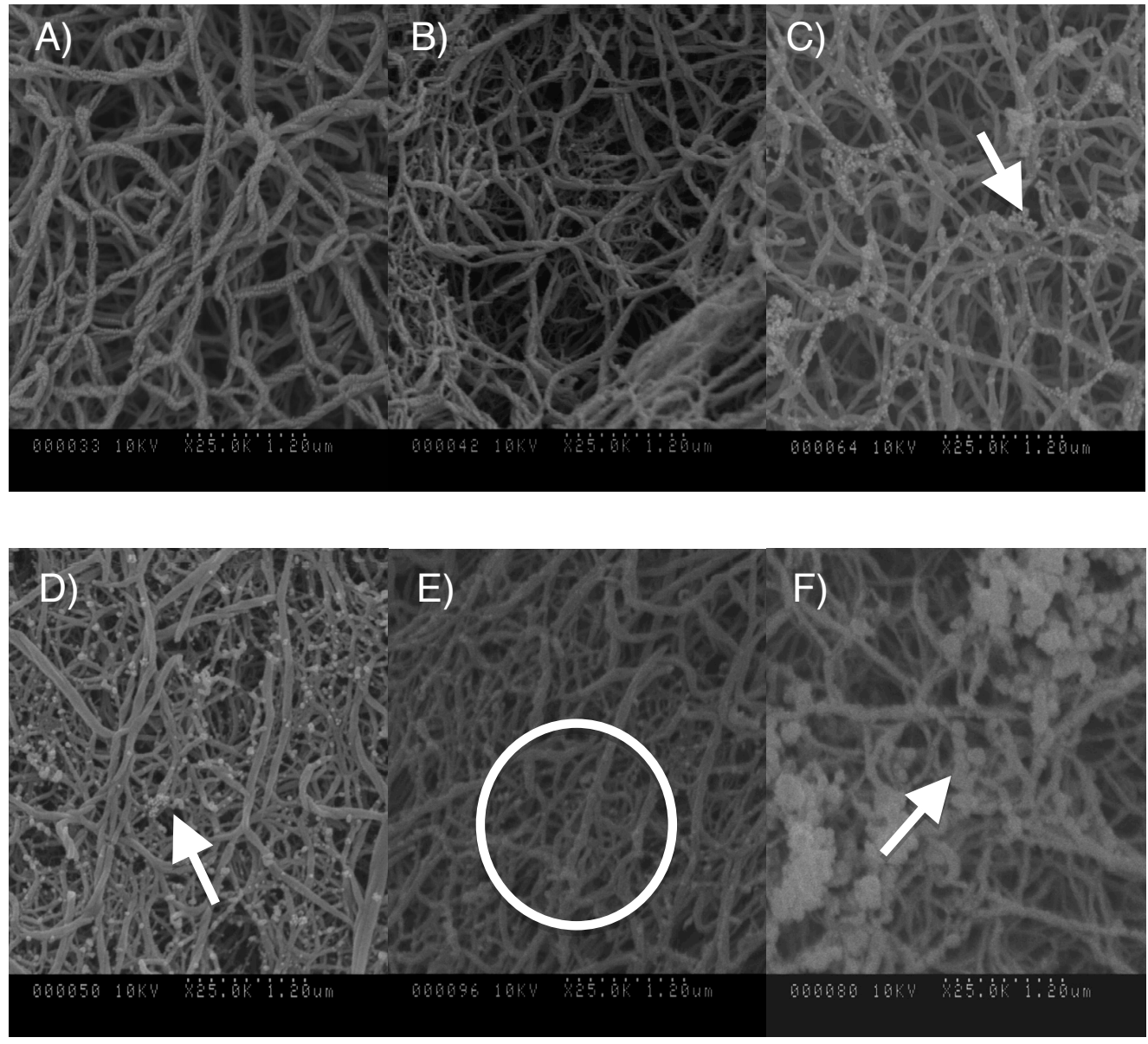


\section{CHAPTER 4}

\section{Discussion}

A discussed earlier, collagen matrix is a major contributor to the viscoelasticity, stability, toughness, and tensile strength of the dentin $(8,23,24)$. Since the integrity of the collagen is maintained by endogenous covalent intermolecular crosslinking of its three helically arranged collagen fibrils (25), disruption of the crosslinkage undermines collagen stability, and leads to alterations in the physical properties of the dentin. An increase in the number of the crosslinks, on the other hand, has the potential to enhance the physical properties and increase dentin resistance to enzymatic degradation.

Collagenase produced by $C$. histolyticum was used in this study. The production of this enzyme by this Gram positive bacteria and other collagenase producing bacteria represents a virulence characteristic that allows them to spread more readily through host tissue, and to acquire small amino acids, derived from collagen degradation, essential to their survival (21). The use of this potent collagenase enzyme represents an aggressive bacterial challenge. The ability to stabilize the collagen substrate against degradation by this enzyme will enable us to overcome the compromise in the dentin micro-structural properties, which results following collagen breakdown. 
The assessment of collagen stability was undertaken by subjecting standardized collagen gels to proteolytic challenge and the use of high concentration of C. histolyticum collagenase may represent an aggressive bacterial challenge (119) as compared to what may occur in vivo, however it did permit us the opportunity to investigate the mechanism of chitosan/collagenase interaction. Bacterial collagenase hydrolyzes the collagen fibril at multiple cleavage sites that correspond with the peptide bond at the amino terminal side of Gly in the Gly-X-Y triple repeat sequence. Collagen is subsequently digested into small peptides (159) and allows the degree of collagen degradation to be quantified by the amount of hydroxyproline released through the digestive action of collagenase. This imino acid, which contains both an imino (>C-NH) and a carboxyl (-C(-O)-OH) group, is unique to collagen and constitutes approximately $10 \%$ of type I collagen mass (160). Pircosirius red stain, a stain specific for the presence of collagen in tissue, while less sensitive than the hydroxyproline assay, was used to confirm enzymatic degradation in the study (161).

Structural changes to the matrix can also result from the use of several endodontic irrigants and disinfectants currently used in root canal treatment. It has been known for some time that varying concentrations of chelating agents, such as EDTA, acids, and disinfecting agents such as sodium hypochlorite $(\mathrm{NaOCl})$, have demonstrated an ability to undermine dentin microhardness and elasticity (60-63), while a more recent study has reported that dentin subject to prolonged calcium hydroxide $(\mathrm{CaOH})$ exposure, demonstrates a decreased resistance to fracture (162). 
The strength of collagen is related to the native crosslinkage of its collagen fibrils. The crosslinks not only improve its mechanical properties, they also offer it greater resistance to enzymatic degradation. Several synthetic and natural chemical agents have been used to increase the number of inter- and intra-molecular collagen crosslinks, in an attempt to increase its mechanical and biological properties $(113,114)$. Glutaraldehyde (GD), a commonly used tissue fixative, is an example of such an agent, and was used as the control for this study. The aldehyde residue of GD reacts with the amino groups of lysyl (Lys) or hydroxylysyl (Hyl) residues in collagen, to produce a network of crosslinks within its structure $(115,117)$. Previous reports have shown that when this occurs, the collagen degradation rate is significantly reduced and the mechanical properties of the dentin preserved $(115,116)$. The disadvantages of using GD as a crosslinking agent in a clinical environment, is the time necessary to affect this change $(\sim 6 \mathrm{~h})$ and its inherent cytotoxicity (113). Shrestha et al. (12) has reported that while GD treatment increases the tensile strength of dentin, it undermined its toughness.

The effectiveness of photodynamic treatment (PDT) as a root canal disinfection protocol has been substantiated by several in vitro and in vivo studies (93, 94, 97, 98, 163, 164). Other studies have shown that the reactive oxygen species produced during PDT not only destroys bacteria, but also interacts with amino acids in the surrounding connective tissue to promote collagen crosslinking (131-133). In this study, FTIR analysis of PDT and GDtreated collagen revealed a reduced amide I peak, relative to the amide II peak. This signaled the conversion of a free $\mathrm{NH}_{2}$ group to $-\mathrm{NH}$ group $(15,165)$ and confirmed formation of additional collagen crosslinks. This in turn could explain the increase in the 
resistance to enzymatic degradation, as demonstrated by hydroxyproline assay, in the PDT and GD treated samples. In a clinical environment this means that PDT has the potential to preserve, and perhaps improve, the mechanical property of dentin undermined by exposure to an infection in which proteolytic bacteria play a part $(12,114$, 166).

The interaction of chitosan and collagen also appears to reduce the rate of collagen degradation (154). The interaction is electrostatic in nature, and may occur, either between the carboxylate groups of collagen $\left(-\mathrm{COO}^{-}\right)$and the ammonium $\left(-\mathrm{NH}_{3}{ }^{+}\right)$ functions of chitosan, or by bonding to hydrogen, depending upon the prevailing conditions (156). In this study, the immunoblotting experiments revealed direct interaction between collagenase and chitosan nanoparticles. Furthermore, the hydroxyproline assay showed that the CSnp-bound collagenase, demonstrated a significant reduction in collagen degradation, when compared to the collagenase in the supernatant with a similar initial concentration. This was a novel finding, and demonstrated that CSnps had an inhibitory effect on collagenolytic activity.

The increased resistance of collagen treated by PDT, CSnp, and PDT+CSnp to collagenase degradation can be interpreted as a potential for affording dentin added resistance to collagenase activity during root canal treatment. Photochemically induced crosslinking may limit the number of hydrolytic sites on the collagen where enzymatic degradation occurs, and thereby inhibit its interaction and subsequent degradation of the 
collagen fibrils. The ability of CSnps to inhibit collagenase, either through direct binding of the nanoparticles to collagenase, as was confirmed by hydroxyproline assay and immunoblotting in this study, or through their inhibition of enzyme recognition of collagen cleavage sites, adds to the valuable potential of PDT and CS as an adjunct to currently accepted treatment protocols. Ultimately its true value will have to be determined in a clinical model where delivery of light and nanoparticles to the dentin of the root canal wall, may prove far more difficult than that associated with their delivery in an in vitro model. However the advantages to be gained by both disinfecting the root canal and preserving or restoring the physical properties of the dentin make the challenge all the more exciting. 


\section{CHAPTER 5}

\section{Conclusions}

Enhancing the resistance of collagen to enzymatic degradation has been proposed as a means of preserving or restoring the integrity of the dentin lost in the course of a root canal infection. To date, studies related to disinfecting irrigants and medicaments used in root canal treatment, have focused primarily on their ability to disrupt and destroy planktonic and biofilm bacteria, while paying little attention to their potential to alter or improve the physical integrity of the dentin. The ability of photodynamic therapy to form crosslinks across exposed collagen fibrils infiltrated with chitosan nanoparticles, may prove to be a novel way of stabilizing and strengthening dentin. Coupled with in vitro studies that have offered encouraging evidence that root canal disinfection can also be effectively accomplished using the same approach $(12,93,94)$ makes it reasonable to suggest that the protocol is worthy of being tested at a higher level.

In summary:

- The resistance of collagen substrate to collagenase degradation is enhanced by pretreatment with PDT, CSnp, and their combination

- The stability of collagen against enzymatic breakdown following the application of PDT was shown to be related to the formation of crosslinks across collagen fibrils

- CSnp infiltrated the collagen network and significantly reduced the activity of collagenase by binding to it 


\section{CHAPTER 6}

\section{Future Studies}

Future studies along this line of research should consider the following recommendations:

- Assess the ultrastructural stability of collagen matrix treated with PDT, CSnp, and their combination following digestion with host-derived MMPs, previously found in native dentin.

- Use human dentin as a source for collagen substrate to assess the resistance to collagenase mediated degradation following pre-treatment with PDT, CSnp, or their combination.

- Study the effect of dentin collagen matrix pre-treatment with PDT, CSnp, or their combination on the mechanical properties of dentin. 


\section{CHAPTER 7}

\section{References}

1. Nair PN. Apical periodontitis: A dynamic encounter between root canal infection and host response. Periodontol 2000. 1997 Feb;13:121-48.

2. Nair PN. On the causes of persistent apical periodontitis: A review. Int Endod J. 2006 Apr;39(4):249-81.

3. Siqueira JF, J., Rocas IN, Paiva SS, Guimaraes-Pinto T, Magalhaes KM, Lima KC. Bacteriologic investigation of the effects of sodium hypochlorite and chlorhexidine during the endodontic treatment of teeth with apical periodontitis. Oral Surg Oral Med Oral Pathol Oral Radiol Endod. 2007 Jul;104(1):122-30.

4. Tang $\mathrm{W}, \mathrm{Wu} \mathrm{Y}$, Smales RJ. Identifying and reducing risks for potential fractures in endodontically treated teeth. J Endod. 2010;36(4):609-17.

5. Sjogren U, Figdor D, Persson S, Sundqvist G. Influence of infection at the time of root filling on the outcome of endodontic treatment of teeth with apical periodontitis. Int Endod J. 1997 Sep;30(5):297-306.

6. Waltimo T, Trope M, Haapasalo M, Orstavik D. Clinical efficacy of treatment procedures in endodontic infection control and one year follow-up of periapical healing. J Endod. 2005 Dec;31(12):863-6.

7. Fabricius L, Dahlen G, Sundqvist G, Happonen RP, Moller AJ. Influence of residual bacteria on periapical tissue healing after chemomechanical treatment and root filling of experimentally infected monkey teeth. Eur J Oral Sci. 2006 Aug;114(4):278-85.

8. Kishen A. Mechanisms and risk factors for fracture predilection in endodontically treated teeth. Endodontic Topics. 2006;13(1):57-83.

9. Niu W, Yoshioka T, Kobayashi C, Suda H. A scanning electron microscopic study of dentinal erosion by final irrigation with EDTA and $\mathrm{NaOCl}$ solutions. Int Endod J. 2002 Nov;35(11):934-9. 
10. Zhang K, Tay FR, Kim YK, Mitchell JK, Kim JR, Carrilho M, et al. The effect of initial irrigation with two different sodium hypochlorite concentrations on the erosion of instrumented radicular dentin. Dent Mater. 2010;26(6):514-23.

11. Kishen A. Advanced therapeutic options for endodontic biofilms. Endodontic Topics. 2010;22(1):99-123.

12. Shrestha A, Friedman S, Kishen A. Photodynamically crosslinked and chitosan-incorporated dentin collagen. J Dent Res. 2011;90(11):1346-51.

13. Rabea EI, Badawy ME, Stevens CV, Smagghe G, Steurbaut W. Chitosan as antimicrobial agent: Applications and mode of action. Biomacromolecules. 2003 Nov-Dec;4(6):1457-65.

14. Kishen A, Shi Z, Shrestha A, Neoh KG. An investigation on the antibacterial and antibiofilm efficacy of cationic nanoparticulates for root canal disinfection. J Endod. 2008 Dec;34(12):151520.

15. Wang XH, Li DP, Wang WJ, Feng QL, Cui FZ, Xu YX, et al. Crosslinked collagen/chitosan matrix for artificial livers. Biomaterials. 2003;24(19):3213-20.

16. Madhavan K, Belchenko D, Motta A, Tan W. Evaluation of composition and crosslinking effects on collagen-based composite constructs. Acta Biomater. 2010;6(4):1413-22.

17. Tjäderhane L, Carrilho MR, Breschi L, Tay FR, Pashley DH. Dentin basic structure and composition-an overview. Endodontic Topics. 2012.

18. Pashley DH. Dynamics of the pulpo-dentin complex. Critical Reviews in Oral Biology and Medicine. 1996;7(2):104-33.

19. Gage JP. Electrophoretic characterization of peptides from normal mature human dentine. Arch Oral Biol. 1984 1984;29(8):575-80.

20. Ramachandran GN, Kartha G. Structure of collagen. Nature. 1955;176(4482):593-5.

21. Harrington DJ. Bacterial collagenases and collagen-degrading enzymes and their potential role in human disease. Infect Immun. 1996;64(6):1885-91. 
22. Bhattacharjee A, Bansal M. Collagen structure: The madras triple helix and the current scenario. IUBMB Life. 2005;57(3):161-72.

23. Miguez PA, Pereira PN, Atsawasuwan P, Yamauchi M. Collagen cross-linking and ultimate tensile strength in dentin. J Dent Res. 2004 Oct;83(10):807-10.

24. Yamauchi M. Collagen Biochemistry: An Overview.Advances in Tissue Banking.Vol.6. 2000:455; 455,500; 500 .

25. Rivera EM, Yamauchi M. Site comparisons of dentine collagen cross-links from extracted human teeth. Arch Oral Biol. 1993;38(7):541-6.

26. Yamauchi M. Collagen Biochemistry: An Overview.Advances in Tissue Banking.Vol.6. 2000:455-500.

27. Slots J, Flynn MJ, Li G. Polymerase chain reaction analysis of the porphyromonas gingivalis collagenase gene. Clinical infectious diseases : an official publication of the Infectious Diseases Society of America. 1995;20 Suppl 2:S167-8.

28. Sundqvist G, Carlsson J, Hanstrom L. Collagenolytic activity of black-pigmented bacteroides species. Journal of periodontal research. 1987;22(4):300-6.

29. Odell LJ, Baumgartner JC, Xia T, David LL. Survey for collagenase gene prtC in porphyromonas gingivalis and porphyromonas endodontalis isolated from endodontic infections. J Endod. 1999;25(8):555-8.

30. Siqueira Jr. JF, Rôças IN. Community as the unit of pathogenicity: An emerging concept as to the microbial pathogenesis of apical periodontitis. Oral Surg Oral Med Oral Pathol Oral Radiol Endod. 2009;107(6):870-8.

31. Ricucci D, Siqueira Jr. JF. Biofilms and apical periodontitis: Study of prevalence and association with clinical and histopathologic findings. J Endod. 2010;36(8):1277-88.

32. Sorsa $T$, Ingman $T$, Suomalainen $K$, Haapasalo $M$, Konttinen $Y T$, Lindy $O$, et al. Identification of proteases from periodontopathogenic bacteria as activators of latent human neutrophil and fibroblast-type interstitial collagenases. Infect Immun. 1992;60(11):4491-5. 
33. Carrilho MR, Tay FR, Donnelly AM, Agee KA, Tjaderhane L, Mazzoni A, et al. Hostderived loss of dentin matrix stiffness associated with solubilization of collagen. Journal of biomedical materials research.Part B, Applied biomaterials. 2009 Jul;90(1):373-80.

34. Matrisian LM. The matrix-degrading metalloproteinases. BioEssays : news and reviews in molecular, cellular and developmental biology. 1992;14(7):455-63.

35. Siqueira Jr. JF. Molecular detection of black-pigmented bacteria in infections of endodontic origin. J Endod. 2001;27(9):563-6.

36. Mayrand D, Grenier D. Detection of collagenase activity in oral bacteria. Canadian journal of microbiology. 1985;31(2):134-8.

37. Steffen EK, Hentges DJ. Hydrolytic enzymes of anaerobic bacteria isolated from human infections. Journal of clinical microbiology. 1981;14(2):153-6.

38. Jin K-, Barua PK, Zambon J, Neiders ME. Proteolytic activity in black-pigmented bacteroides species. J Endod. 1989;15(10):463-7.

39. Keudell K, Conte M. Enzyme of microbial isolates from infected pulp chambers--a preliminary report. Journal of endodontics. 1976;2(7):217-9.

40. Gold OG, Jordan HV, van Houte J. The prevalence of enterococci in the human mouth and their pathogenicity in animal models. Archives of oral biology. 1975;20(7):473-7.

41. Robertson PB, Cobb CM, Taylor RE, Fullmer HM. Activation of latent collagenase by microbial plaque. Journal of periodontal research. 1974;9(2):81-3.

42. Hannas AR, Pereira JC, Granjeiro JM, Tjaderhane L. The role of matrix metalloproteinases in the oral environment. Acta Odontol Scand. 2007 Feb;65(1):1-13.

43. Geiger SB, Harper E. The inhibition of human gingival collagenase by an inhibitor extracted from human teeth. J Periodont Res. 1981 Jan;16(1):8-12.

44. Goldberg M, Septier D, Bourd K, Hall R, George A, Goldberg H, et al. Immunohistochemical localization of MMP-2, MMP-9, TIMP-1, and TIMP-2 in the forming rat incisor. Connect Tissue Res. 2003;44(3-4):143-53. 
45. Martin-De LH, Valenzuela A, Overall CM. The matrix metalloproteinase gelatinase A in human dentine. Arch Oral Biol. 2000 Sep;45(9):757-65.

46. Santos J, Carrilho M, Tervahartiala T, Sorsa T, Breschi L, Mazzoni A, et al. Determination of matrix metalloproteinases in human radicular dentin. J Endod. 2009 May;35(5):686-9.

47. Sulkala M, Tervahartiala $T$, Sorsa $T$, Larmas $M$, Salo $T$, Tjaderhane L. Matrix metalloproteinase-8 (MMP-8) is the major collagenase in human dentin. Arch Oral Biol. 2007 Feb;52(2):121-7.

48. Sulkala M, Larmas M, Sorsa $T$, Salo $T$, Tjaderhane L. The localization of matrix metalloproteinase-20 (MMP-20, enamelysin) in mature human teeth. J Dent Res. 2002 Sep;81(9):603-7.

49. Barkhordar RA. Determining the presence and origin of collagenase in human periapical lesions. J Endod. 1987;13(5):228-32.

50. Tjaderhane L, Larjava H, Sorsa T, Uitto VJ, Larmas M, Salo T. The activation and function of host matrix metalloproteinases in dentin matrix breakdown in caries lesions. J Dent Res. 1998 Aug;77(8):1622-9.

51. van Strijp AJ, Jansen DC, DeGroot J, ten Cate JM, Everts V. Host-derived proteinases and degradation of dentine collagen in situ. Caries Res. 2003 Jan-Feb;37(1):58-65.

52. Chaussain-Miller C, Fioretti F, Goldberg M, Menashi S. The role of matrix metalloproteinases (MMPs) in human caries. J Dent Res. 2006 Jan;85(1):22-32.

53. Reynaud aG, Sorsa T, Stackelberg S, Tervahartiala T, Haapasalo M. Effect of E. faecalis on the release of serine proteases elastase and cathepsin G, and collagenase-2 (MMP-8) by human polymorphonuclear leukocytes (PMNs). Int Endod J. 2005 Sep;38(9):667-77.

54. Vier FV, Figueiredo JA. Internal apical resorption and its correlation with the type of apical lesion. Int Endod J. 2004 Nov;37(11):730-7.

55. Ingman $\mathrm{T}$, Tervahartiala $\mathrm{T}$, Ding $\mathrm{Y}$, Tschesche $\mathrm{H}$, Haerian A, Kinane DF, et al. Matrix metalloproteinases and their inhibitors in gingival crevicular fluid and saliva of periodontitis patients. Journal of clinical periodontology. 1996;23(12):1127-32. 
56. Rai B, Kharb S, Jain R, Anand SC. Biomarkers of periodontitis in oral fluids. Journal of oral science. 2008;50(1):53-6.

57. Furuholm J, Sorsa T, Qvarnstrom M, Janket SJ, Tervahartiala T, Nuutinen P, et al. Salivary matrix metalloproteinase- 8 in patients with and without coronary heart disease may indicate an increased susceptibility to periodontal disease. Journal of periodontal research. 2006;41(5):486-9.

58. Isaza-Guzmán DM, Arias-Osorio C, Martínez-Pabón MC, Tobón-Arroyave SI. Salivary levels of matrix metalloproteinase (MMP)-9 and tissue inhibitor of matrix metalloproteinase (TIMP)-1: A pilot study about the relationship with periodontal status and MMP-9-1562C/T gene promoter polymorphism. Arch Oral Biol. 2011;56(4):401-11.

59. Qian W, Shen Y, Haapasalo M. Quantitative analysis of the effect of irrigant solution sequences on dentin erosion. J Endod. 2011;37(10):1437-41.

60. Saleh AA, Ettman WM. Effect of endodontic irrigation solutions on microhardness of root canal dentine. Journal of dentistry. 1999;27(1):43-6.

61. Sayin TC, Serper A, Cehreli ZC, Otlu HG. The effect of EDTA, EGTA, EDTAC, and tetracycline- $\mathrm{HCl}$ with and without subsequent $\mathrm{NaOCl}$ treatment on the microhardness of root canal dentin. Oral surgery, oral medicine, oral pathology, oral radiology, and endodontics. 2007;104(3):418-24.

62. Sim TP, Knowles JC, Ng YL, Shelton J, Gulabivala K. Effect of sodium hypochlorite on mechanical properties of dentine and tooth surface strain. International endodontic journal. 2001;34(2):120-32.

63. Baron M, Llena C, Forner L, Palomares M, Gonzalez-Garcia C, Salmeron-Sanchez M. Nanostructural changes in dentine caused by endodontic irrigants. Med Oral Patol Oral Cir Bucal. 2013 Jul 1;18(4):e733-6.

64. Ferrari M, Mason PN, Goracci C, Pashley DH, Tay FR. Collagen degradation in endodontically treated teeth after clinical function. J Dent Res. 2004 May;83(5):414-9.

65. Mazzoni A, Pashley DH, Nishitani Y, Breschi L, Mannello F, Tjaderhane L, et al. Reactivation of inactivated endogenous proteolytic activities in phosphoric acid-etched dentine by etch-and-rinse adhesives. Biomaterials. 2006 Sep;27(25):4470-6. 
66. Nishitani Y, Yoshiyama M, Wadgaonkar B, Breschi L, Mannello F, Mazzoni A, et al. Activation of gelatinolytic/collagenolytic activity in dentin by self-etching adhesives. Eur J Oral Sci. 2006 Apr;114(2):160-6.

67. Dai T, Huang YY, Hamblin MR. Photodynamic therapy for localized infections--state of the art. Photodiagnosis and photodynamic therapy. 2009;6(3-4):170-88.

68. Konopka K, Goslinski T. Photodynamic therapy in dentistry. J Dent Res. 2007 Aug;86(8):694-707.

69. Souza LC, Brito PR, de Oliveira JC, Alves FR, Moreira EJ, Sampaio-Filho H, et al. Photodynamic therapy with two different photosensitizers as a supplement to instrumentation/irrigation procedures in promoting intracanal reduction of enterococcus faecalis. J Endod Feb;36(2):292-6.

70. Jori G, Fabris C, Soncin M, Ferro S, Coppellotti O, Dei D, et al. Photodynamic therapy in the treatment of microbial infections: Basic principles and perspective applications. Lasers Surg Med. 2006 Jun;38(5):468-81.

71. Hamblin MR, Hasan T. Photodynamic therapy: A new antimicrobial approach to infectious disease? Photochemical \& photobiological sciences : Official journal of the European Photochemistry Association and the European Society for Photobiology. 2004 May;3(5):436-50.

72. George S, Kishen A. Influence of photosensitizer solvent on the mechanisms of photoactivated killing of enterococcus faecalis. Photochem Photobiol. 2008 May-Jun;84(3):73440.

73. Bertoloni G, Lauro FM, Cortella G, Merchat M. Photosensitizing activity of hematoporphyrin on staphylococcus aureus cells. Biochimica et biophysica acta. 2000;1475(2):169-74.

74. Menezes S, Capella MA, Caldas LR. Photodynamic action of methylene blue: Repair and mutation in escherichia coli. Journal of photochemistry and photobiology B, Biology. 1990;5(34):505-17.

75. Moan J, Berg K. The photodegradation of porphyrins in cells can be used to estimate the lifetime of singlet oxygen. Photochemistry and photobiology. 1991;53(4):549-53. 
76. Shen HR, Spikes JD, Kopecekova P, Kopecek J. Photodynamic crosslinking of proteins. I. model studies using histidine- and lysine-containing N-(2-hydroxypropyl)methacrylamide copolymers. Journal of photochemistry and photobiology B, Biology. 1996;34(2-3):203-10.

77. Nitzan Y, Gutterman M, Malik Z, Ehrenberg B. Inactivation of gram-negative bacteria by photosensitized porphyrins. Photochemistry and photobiology. 1992;55(1):89-96.

78. Upadya MH, Kishen A. Influence of bacterial growth modes on the susceptibility to lightactivated disinfection. Int Endod J. 2010;43(11):978-87.

79. Denyer SP, Maillard JY. Cellular impermeability and uptake of biocides and antibiotics in gram-negative bacteria. Journal of applied microbiology. 2002;92 Suppl:35s-45s.

80. Wilson M, Burns T, Pratten J, Pearson GJ. Bacteria in supragingival plaque samples can be killed by low-power laser light in the presence of a photosensitizer. The Journal of applied bacteriology. 1995;78(5):569-74.

81. Merchat M, Bertolini G, Giacomini P, Villanueva A, Jori G. Meso-substituted cationic porphyrins as efficient photosensitizers of gram-positive and gram-negative bacteria. Journal of photochemistry and photobiology B, Biology. 1996;32(3):153-7.

82. Ryan BM, Dougherty TJ, Beaulieu D, Chuang J, Dougherty BA, Barrett JF. Efflux in bacteria: What do we really know about it? Expert opinion on investigational drugs. 2001;10(8):1409-22.

83. Zhang L, Mah TF. Involvement of a novel efflux system in biofilm-specific resistance to antibiotics. Journal of bacteriology. 2008;190(13):4447-52.

84. Kvist M, Hancock V, Klemm P. Inactivation of efflux pumps abolishes bacterial biofilm formation. Applied and environmental microbiology. 2008;74(23):7376-82.

85. Tegos GP, Hamblin MR. Phenothiazinium antimicrobial photosensitizers are substrates of bacterial multidrug resistance pumps. Antimicrobial agents and chemotherapy. 2006;50(1):196203.

86. Tegos GP, Masago K, Aziz F, Higginbotham A, Stermitz FR, Hamblin MR. Inhibitors of bacterial multidrug efflux pumps potentiate antimicrobial photoinactivation. Antimicrobial agents and chemotherapy. 2008;52(9):3202-9. 
87. Kishen A, Upadya M, Tegos GP, Hamblin MR. Efflux pump inhibitor potentiates antimicrobial photodynamic inactivation of enterococcus faecalis biofilm. Photochem Photobiol. 2010;86(6):1343-9.

88. Upadya M, Shrestha A, Kishen A. Role of efflux pump inhibitors on the antibiofilm efficacy of calcium hydroxide, chitosan nanoparticles, and light-activated disinfection. $\mathrm{J}$ Endod. 2011;37(10):1422-6.

89. Fimple JL, Fontana CR, Foschi F, Ruggiero K, Song X, Pagonis TC, et al. Photodynamic treatment of endodontic polymicrobial infection in vitro. J Endod. 2008 Jun;34(6):728-34.

90. George S, Kishen A. Augmenting the antibiofilm efficacy of advanced noninvasive light activated disinfection with emulsified oxidizer and oxygen carrier. J Endod. 2008 Sep;34(9):1119-23.

91. Lima JP, Sampaio dM, Borges FM, Teixeira AH, Steiner-Oliveira C, Nobre DS, et al. Evaluation of the antimicrobial effect of photodynamic antimicrobial therapy in an in situ model of dentine caries. European journal of oral sciences. 2009;117(5):568-74.

92. Molander A, Reit C, Dahlén G, Kvist T. Microbiological status of root-filled teeth with apical periodontitis. Int Endod J. 1998;31(1):1-7.

93. Soukos NS, Chen PS, Morris JT, Ruggiero K, Abernethy AD, Som S, et al. Photodynamic therapy for endodontic disinfection. J Endod. 2006 Oct;32(10):979-84.

94. Williams JA, Pearson GJ, Colles MJ. Antibacterial action of photoactivated disinfection $\{P A D\}$ used on endodontic bacteria in planktonic suspension and in artificial and human root canals. J Dent. 2006 Jul;34(6):363-71.

95. George S, Kishen A. Advanced noninvasive light-activated disinfection: Assessment of cytotoxicity on fibroblast versus antimicrobial activity against enterococcus faecalis. Journal of endodontics. 2007;33(5):599-602.

96. Meire MA, De Prijck K, Coenye T, Nelis HJ, De Moor RJ. Effectiveness of different laser systems to kill enterococcus faecalis in aqueous suspension and in an infected tooth model. Int Endod J. 2009 Apr;42(4):351-9. 
97. Bonsor SJ, Nichol R, Reid TM, Pearson GJ. An alternative regimen for root canal disinfection. Br Dent J. 2006 Jul 22;201(2):101,5; discussion 98; quiz 120.

98. Garcez AS, Nunez SC, Hamblin MR, Ribeiro MS. Antimicrobial effects of photodynamic therapy on patients with necrotic pulps and periapical lesion. J Endod. 2008 Feb;34(2):138-42.

99. Garcez AS, Nuñez SC, Hamblim MR, Suzuki H, Ribeiro MS. Photodynamic therapy associated with conventional endodontic treatment in patients with antibiotic-resistant microflora: A preliminary report. J Endod. 2010;36(9):1463-6.

100. Haapasalo M, Shen Y. Current therapeutic options for endodontic biofilms. Endodontic Topics. 2010;22(1):79-98.

101. Soukos NS, Wilson M, Burns T, Speight PM. Photodynamic effects of toluidine blue on human oral keratinocytes and fibroblasts and streptococcus sanguis evaluated in vitro. Lasers in surgery and medicine. 1996;18(3):253-9.

102. Xu Y, Young MJ, Battaglino RA, Morse LR, Fontana CR, Pagonis TC, et al. Endodontic antimicrobial photodynamic therapy: Safety assessment in mammalian cell cultures. J Endod. 2009 Nov;35(11):1567-72.

103. Soncin M, Fabris C, Busetti A, Dei D, Nistri D, Roncucci G, et al. Approaches to selectivity in the zn(II)-phthalocyanine-photosensitized inactivation of wild-type and antibiotic-resistant staphylococcus aureus. Photochemical \& photobiological sciences : Official journal of the European Photochemistry Association and the European Society for Photobiology. 2002;1(10):815-9.

104. Jang WD, Nakagishi Y, Nishiyama N, Kawauchi S, Morimoto Y, Kikuchi M, et al. Polyion complex micelles for photodynamic therapy: Incorporation of dendritic photosensitizer excitable at long wavelength relevant to improved tissue-penetrating property. Journal of controlled release : official journal of the Controlled Release Society. 2006;113(1):73-9.

105. George S, Kishen A. Photophysical, photochemical, and photobiological characterization of methylene blue formulations for light-activated root canal disinfection. J Biomed Opt. $2007 ; 12(3)$. 
106. Soukos NS, Hamblin MR, Hasan T. The effect of charge on cellular uptake and phototoxicity of polylysine chlorin(e6) conjugates. Photochemistry and photobiology. 1997;65(4):723-9.

107. Hamblin MR, O'Donnell DA, Murthy N, Rajagopalan K, Michaud N, Sherwood ME, et al. Polycationic photosensitizer conjugates: Effects of chain length and gram classification on the photodynamic inactivation of bacteria. The Journal of antimicrobial chemotherapy. 2002;49(6):941-51.

108. Bezman SA, Burtis PA, Izod TP, Thayer MA. Photodynamic inactivation of E. coli by rose bengal immobilized on polystyrene beads. Photochemistry and photobiology. 1978;28(3):325-9.

109. Friedberg JS, Tompkins RG, Rakestraw SL, Warren SW, Fischman AJ, Yarmush ML. Antibody-targeted photolysis. bacteriocidal effects of sn (IV) chlorin e6-dextran-monoclonal antibody conjugates. Annals of the New York Academy of Sciences. 1991;618:383-93.

110. Gross S, Brandis A, Chen L, Rosenbach-Belkin V, Roehrs S, Scherz A, et al. Protein-Amediated targeting of bacteriochlorophyll-IgG to staphylococcus aureus: A model for enhanced site-specific photocytotoxicity. Photochemistry and photobiology. 1997;66(6):872-8.

111. Klepac-Ceraj V, Patel N, Song X, Holewa C, Patel C, Kent R, et al. Photodynamic effects of methylene blue-loaded polymeric nanoparticles on dental plaque bacteria. Lasers Surg Med. 2011;43(7):600-6.

112. Pagonis TC, Chen J, Fontana CR, Devalapally H, Ruggiero K, Song X, et al. Nanoparticlebased endodontic antimicrobial photodynamic therapy. J Endod. 2010;36(2):322-8.

113. Han B, Jaurequi J, Tang BW, Nimni ME. Proanthocyanidin: A natural crosslinking reagent for stabilizing collagen matrices. Journal of biomedical materials research Part A. 2003;65(1):118-24.

114. Bedran-Russo A, Pereira PN, Duarte WR, Drummond JL, Yamauchi M. Application of crosslinkers to dentin collagen enhances the ultimate tensile strength. Journal of biomedical materials research.Part B, Applied biomaterials. 2007 Jan;80(1):268-72. 
115. Sung HW, Huang DM, Chang WH, Huang RN, Hsu JC. Evaluation of gelatin hydrogel crosslinked with various crosslinking agents as bioadhesives: In vitro study. Journal of biomedical materials research. 1999;46(4):520-30.

116. Nimni ME. Bioprosthesis derived from crosslinked and chemically modified collagenous tissues. Nimni ME. 1988:1; 1,38; 38.

117. Ritter AV, Swift EJ, J., Yamauchi M. Effects of phosphoric acid and glutaraldehyde-HEMA on dentin collagen. European journal of oral sciences. 2001;109(5):348-53.

118. Bedran-Russo A, Pashley DH, Agee K, Drummond JL, Miescke KJ. Changes in stiffness of demineralized dentin following application of collagen crosslinkers. Journal of biomedical materials research.Part B, Applied biomaterials. 2008 Aug;86B(2):330-4.

119. Bedran-Russo A, Yoo KJ, Ema KC, Pashley DH. Mechanical properties of tannic-acidtreated dentin matrix. J Dent Res. 2009 Sep;88(9):807-11.

120. Hebling J, Pashley DH, Tjaderhane L, Tay FR. Chlorhexidine arrests subclinical degradation of dentin hybrid layers in vivo. J Dent Res. 2005 Aug;84(8):741-6.

121. Carrilho MR, Geraldeli S, Tay F, de Goes MF, Carvalho RM, Tjaderhane L, et al. In vivo preservation of the hybrid layer by chlorhexidine. J Dent Res. 2007 Jun;86(6):529-33.

122. Pashley DH, Tay FR, Yiu C, Hashimoto M, Breschi L, Carvalho RM, et al. Collagen degradation by host-derived enzymes during aging. J Dent Res. 2004 Mar;83(3):216-21.

123. Tay FR, Pashley DH, Loushine RJ, Weller RN, Monticelli F, Osorio R. Self-etching adhesives increase collagenolytic activity in radicular dentin. J Endod. 2006 Sep;32(9):862-8.

124. Gendron R, Grenier D, Sorsa T, Mayrand D. Inhibition of the activities of matrix metalloproteinases 2, 8, and 9 by chlorhexidine. Clin Diagn Lab Immunol. 1999 May;6(3):437-9.

125. Zheng X, Hu J, Chen Y, Zhu Y, Chen H. AFM study of the effects of collagenase and its inhibitors on dentine collagen fibrils. J Dent. 2012;40(2):163-71.

126. Basrani BR, Manek S, Sodhi RN, Fillery E, Manzur A. Interaction between sodium hypochlorite and chlorhexidine gluconate. J Endod. 2007 Aug;33(8):966-9. 
127. Prado M, Santos Junior HM, Rezende CM, Pinto AC, Faria RB, Simao RA, et al. Interactions between irrigants commonly used in endodontic practice: A chemical analysis. $\mathrm{J}$ Endod. 2013 Apr;39(4):505-10.

128. Akisue E, Tomita VS, Gavini G, Poli de Figueiredo JA. Effect of the combination of sodium hypochlorite and chlorhexidine on dentinal permeability and scanning electron microscopy precipitate observation. J Endod. 2010 May;36(5):847-50.

129. Bui TB, Baumgartner JC, Mitchell JC. Evaluation of the interaction between sodium hypochlorite and chlorhexidine gluconate and its effect on root dentin. J Endod. 2008 Feb;34(2):181-5.

130. Vivacqua-Gomes N, Ferraz CC, Gomes BP, Zaia AA, Teixeira FB, Souza-Filho FJ. Influence of irrigants on the coronal microleakage of laterally condensed gutta-percha root fillings. Int Endod J. 2002 Sep;35(9):791-5.

131. Chan BP, Chan OC, So KF. Effects of photochemical crosslinking on the microstructure of collagen and a feasibility study on controlled protein release. Acta biomaterialia. 2008 Nov;4(6):1627-36.

132. Spikes JD, Shen H-, Kopecková P, Kopecek J. Photodynamic crosslinking of proteins. III. kinetics of the FMN- and rose bengal-sensitized photooxidation and intermolecular crosslinking of model tyrosine-containing N-(2-hydroxypropyl)methacrylamide copolymers. Photochem Photobiol. 1999;70(2):130-7.

133. Wollensak G, Iomdina E. Long-term biomechanical properties of rabbit cornea after photodynamic collagen crosslinking. Acta Ophthalmol. 2009;87(1):48-51.

134. Breschi L, Mazzoni A, Nato F, Carrilho M, Visintini E, Tjäderhane L, et al. Chlorhexidine stabilizes the adhesive interface: A 2-year in vitro study. Dent Mater. 2010;26(4):320-5.

135. Kochevar IE, Redmond RW. Photosensitized production of singlet oxygen. Methods in enzymology. 2000;319:20-8.

136. Chan BP, So KF. Photochemical crosslinking improves the physicochemical properties of collagen scaffolds. Journal of biomedical materials research Part A. 2005;75(3):689-701. 
137. Shi Z, Neoh KG, Kang ET, Wang W. Antibacterial and mechanical properties of bone cement impregnated with chitosan nanoparticles. Biomaterials. 2006 Apr;27(11):2440-9.

138. Cuero RG, Duffus E, Osuji G, Pettit R. Aflatoxin control in preharvest maize: Effects of chitosan and two microbial agents. Journal of Agricultural Science (Cambridge). 1991;117(2):165-9.

139. Sudarshan NR, Hoover DG, Knorr D. Antibacterial action of chitosan. Food Biotechnol. 1992;6(3):257-72.

140. Muzzarelli R, Tarsi R, Filippini O, Giovanetti E, Biagini G, Varaldo PE. Antimicrobial properties of N-carboxybutyl chitosan. Antimicrobial Agents Chemother. 1990;34(10):2019-23.

141. Kumar MN, Muzzarelli RA, Muzzarelli C, Sashiwa H, Domb AJ. Chitosan chemistry and pharmaceutical perspectives. Chem Rev. 2004 Dec;104(12):6017-84.

142. Yoon KY, Hoon Byeon J, Park JH, Hwang J. Susceptibility constants of escherichia coli and bacillus subtilis to silver and copper nanoparticles. The Science of the total environment. 2007;373(2-3):572-5.

143. Reddy KM, Feris K, Bell J, Wingett DG, Hanley C, Punnoose A. Selective toxicity of zinc oxide nanoparticles to prokaryotic and eukaryotic systems. Applied physics letters. 2007;90(213902):2139021-3.

144. Cioffi N, Ditaranto N, Torsi L, Picca RA, Sabbatini L, Valentini A, et al. Analytical characterization of bioactive fluoropolymer ultra-thin coatings modified by copper nanoparticles. Analytical and bioanalytical chemistry. 2005;381(3):607-16.

145. Nweke CO, Alisi CS, Okolo JC, Nwanyanwu CE. Toxicity of zinc to heterotrophic bacteria from a tropical river sediment. Appl Ecol Environ Res. 2007;5(1):123-32.

146. Beard SJ, Hughes MN, Poole RK. Inhibition of the cytochrome bd-terminated NADH oxidase system in escherichia coli $\mathrm{K}-12$ by divalent metal cations. FEMS microbiology letters. 1995;131(2):205-10.

147. Feng QL, Wu J, Chen GQ, Cui FZ, Kim TN, Kim JO. A mechanistic study of the antibacterial effect of silver ions on escherichia coli and staphylococcus aureus. Journal of biomedical materials research. 2000;52(4):662-8. 
148. Kim JS, Kuk E, Yu KN, Kim JH, Park SJ, Lee HJ, et al. Antimicrobial effects of silver nanoparticles. Nanomedicine : nanotechnology, biology, and medicine. 2007;3(1):95-101.

149. Sawai J. Quantitative evaluation of antibacterial activities of metallic oxide powders ( $\mathrm{ZnO}$, $\mathrm{MgO}$ and $\mathrm{CaO}$ ) by conductimetric assay. Journal of microbiological methods. 2003;54(2):177-82.

150. Yamamoto O. Influence of particle size on the antibacterial activity of zinc oxide. Int J Inorg Mater. 2001;3(7):643-6.

151. Shrestha A, Zhilong S, Gee NK, Kishen A. Nanoparticulates for antibiofilm treatment and effect of aging on its antibacterial activity. J Endod. 2010;36(6):1030-5.

152. Habelitz S, Balooch M, Marshall SJ, Balooch G, Marshall GW, J. In situ atomic force microscopy of partially demineralized human dentin collagen fibrils. Journal of structural biology. 2002;138(3):227-36.

153. Kishen A, Sum CP, Mathew S, Lim CT. Influence of irrigation regimens on the adherence of enterococcus faecalis to root canal dentin. J Endod. 2008 Jul;34(7):850-4.

154. Chen J, Li Q, Xu J, Huang Y, Ding Y, Deng H, et al. Study on biocompatibility of complexes of collagen-chitosan-sodium hyaluronate and cornea. Artif Organs. 2005 Feb;29(2):104-13.

155. Taravel MN, Domard A. Collagen and its interactions with chitosan, III some biological and mechanical properties. Biomaterials. 1996 Feb;17(4):451-5.

156. Taravel MN, Domard A. Relation between the physicochemical characteristics of collagen and its interactions with chitosan: I. Biomaterials. 1993 Oct;14(12):930-8.

157. Gage JP. Electrophoretic characterization of peptides from normal mature human dentine. Archives of oral biology. 1984;29(8):575-80.

158. Mayrand D, Grenier D. Detection of collagenase activity in oral bacteria. Can J Microbiol. $1985 ; 31(2): 134-8$.

159. Supuran CT, Scozzafava A, Clare BW. Bacterial protease inhibitors. Medicinal research reviews. 2002;22(4):329-72. 
160. Bornstein P, Sage H. Structurally distinct collagen types. Annual review of biochemistry. 1980;49:957-1003.

161. Junqueira LC, Bignolas G, Brentani RR. Picrosirius staining plus polarization microscopy, a specific method for collagen detection in tissue sections. The Histochemical journal. 1979;11(4):447-55.

162. Zarei M, Afkhami F, Malek Poor Z. Fracture resistance of human root dentin exposed to calcium hydroxide intervisit medication at various time periods: An in vitro study. Dent Traumatol. 2013;29(2):156-60.

163. Bergmans L, Moisiadis P, Huybrechts B, Van Meerbeek B, Quirynen M, Lambrechts P. Effect of photo-activated disinfection on endodontic pathogens ex vivo. Int Endod J. 2008 Mar;41(3):227-39.

164. Lim Z, Cheng JL, Lim TW, Teo EG, Wong J, George S, et al. Light activated disinfection: An alternative endodontic disinfection strategy. Aust Dent J. 2009 Jun;54(2):108-14.

165. Rafat M, Li F, Fagerholm P, Lagali NS, Watsky MA, Munger R, et al. PEG-stabilized carbodiimide crosslinked collagen-chitosan hydrogels for corneal tissue engineering. Biomaterials. 2008;29(29):3960-72.

166. Breschi L, Mazzoni A, Nato F, Carrilho M, Visintini E, Tjaderhane L, et al. Chlorhexidine stabilizes the adhesive interface: A 2-year in vitro study. Dental materials : official publication of the Academy of Dental Materials Apr;26(4):320-5. 


\section{Appendices}

\section{Appendix 1}

\section{Chemical Structures}

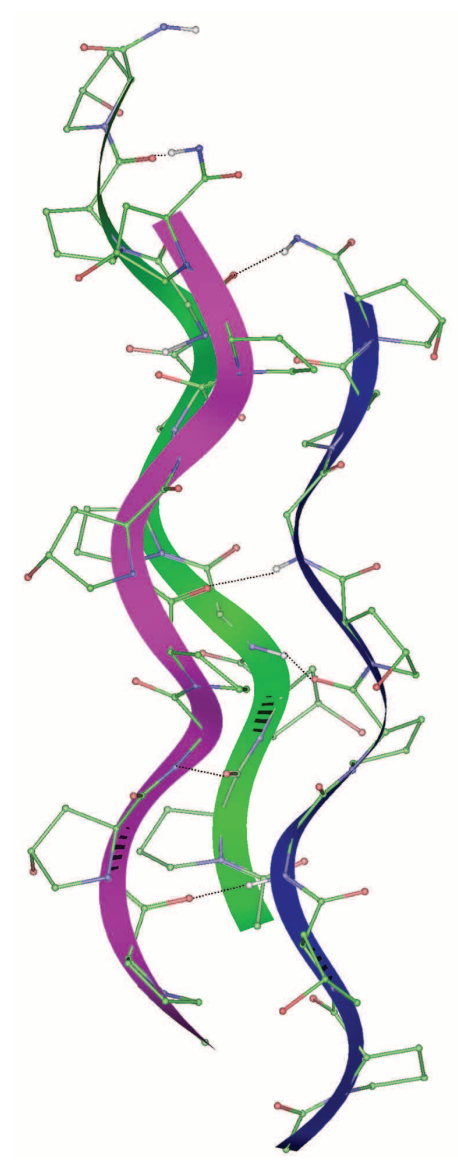

Figure 1. Structure of collagen. Ball and stick diagrams showing two projections of the currently accepted triple helical structure for collagen with one inter-chain hydrogen bond per tripeptide. The sequence shown is (Gly-Pro-Hyp $)_{3}$ and each chain in the triple helix has a different color ribbon drawn through the backbone. (Adapted from Bhattacharjee \& Bansal, 2005) 


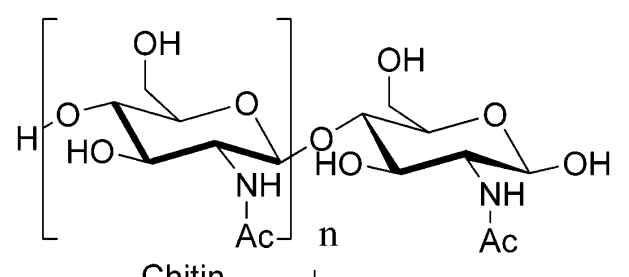

Chitin
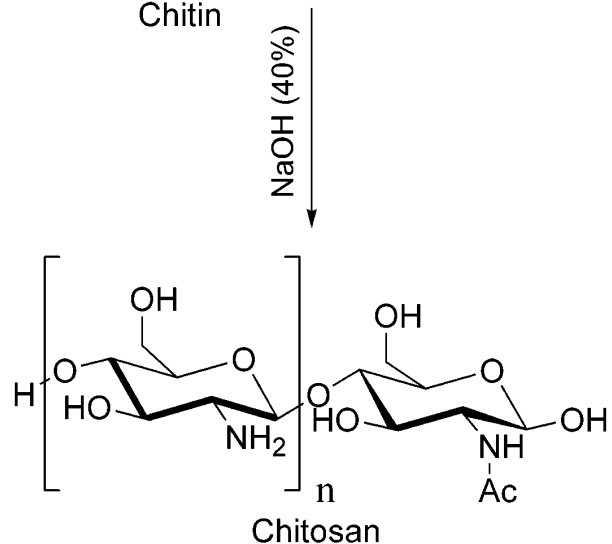

Figure 2. Preparation of chitosan from chitin. Chitosan is a polycationic biopolymer and contains more than 5000 glucosamine units. It is derived from alkaline deacetylation of chitin that is obtained from the shells of crustaceans. (Adapted from Rabea et al., 2003) 


\section{Appendix 2}

\section{Preliminary Data}

A)

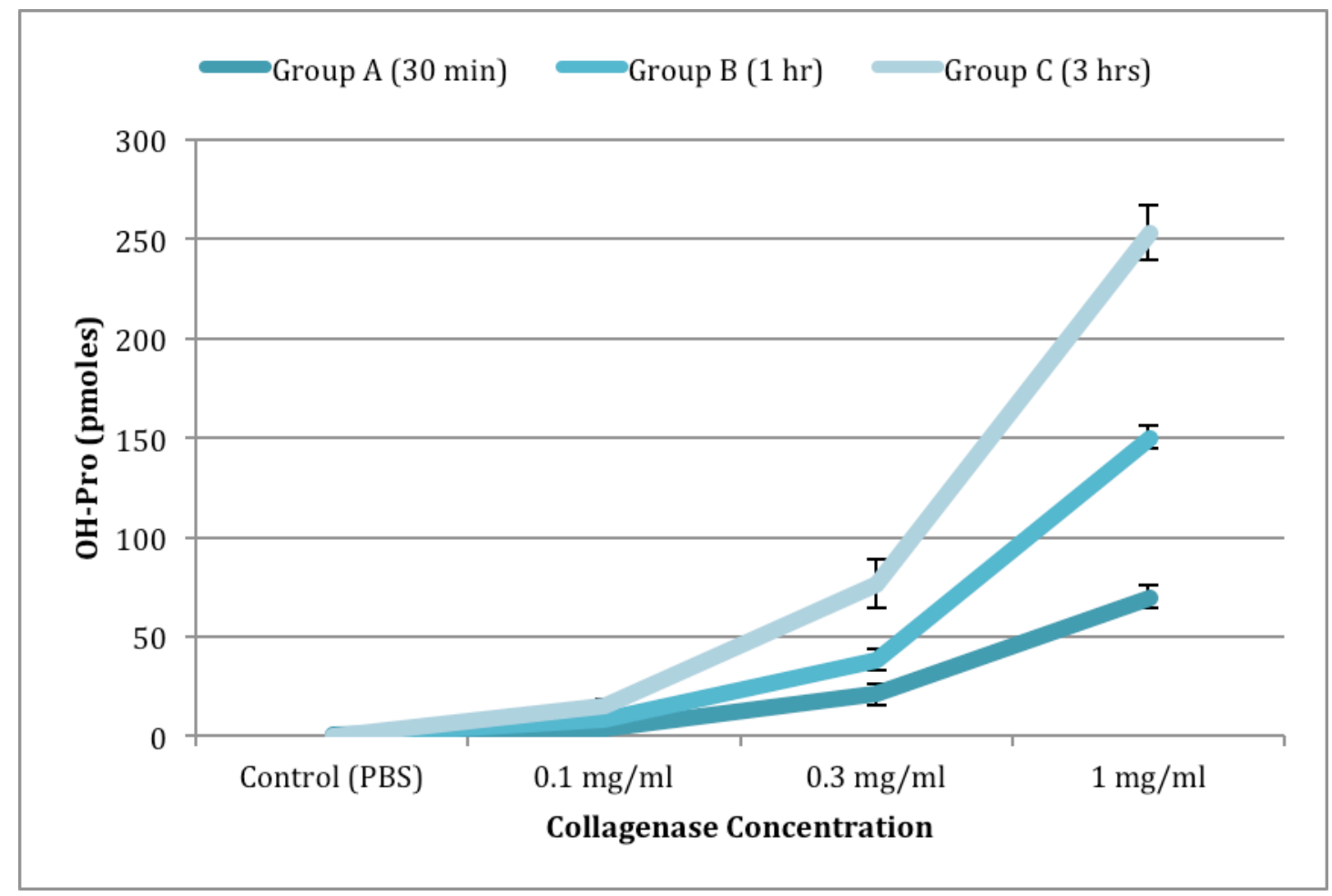


B)

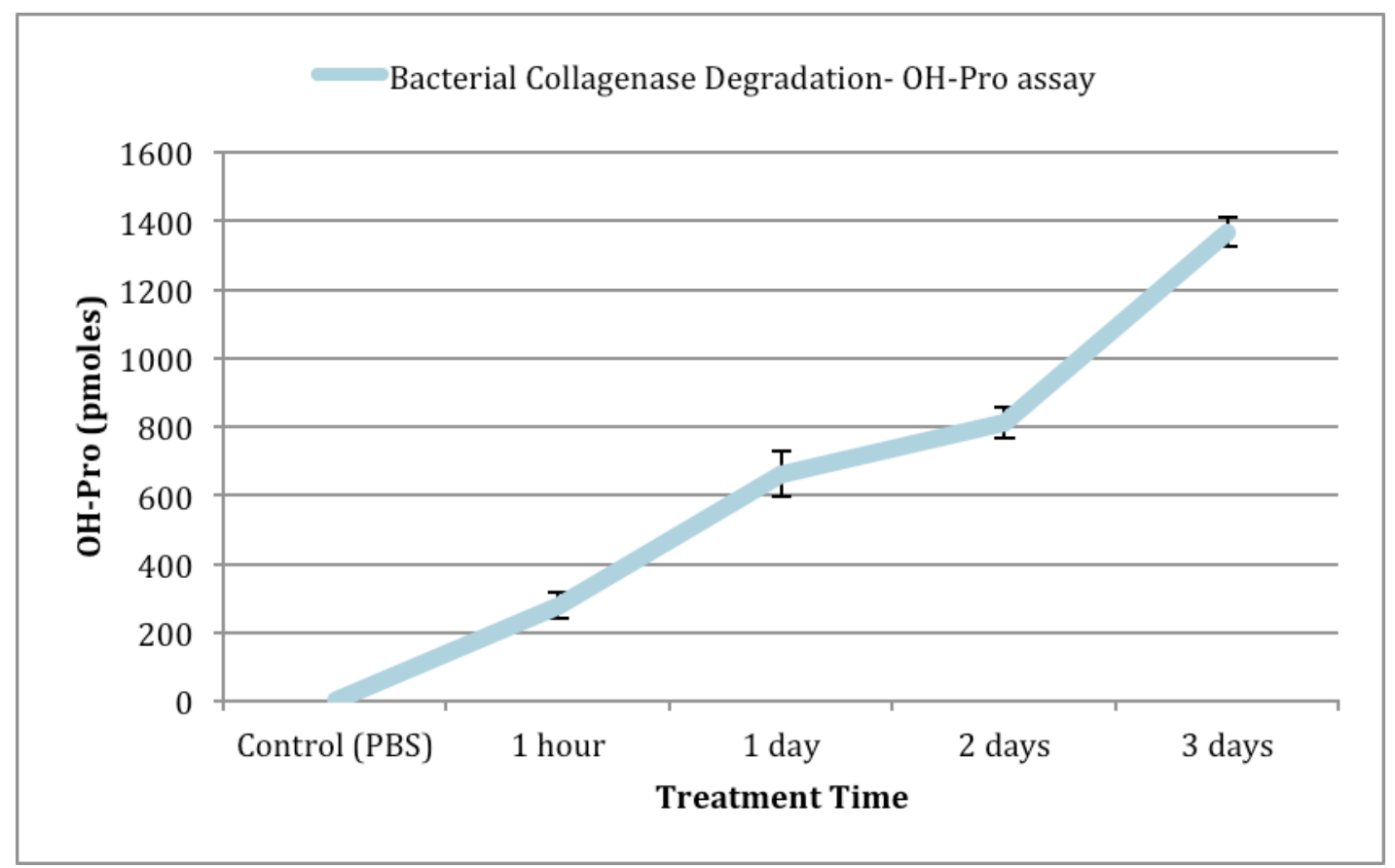

Figure 3. Hydroxyproline Assays. A) Mean concentration of OH-Pro released after collagen gel degradation with $C$. histolyticum at various times and concentrations. B) Collagen gel degradation with $1 \mathrm{mg} / \mathrm{ml}$ bacterial collagenase for 1 hour, 1,2 , and 3 days. 


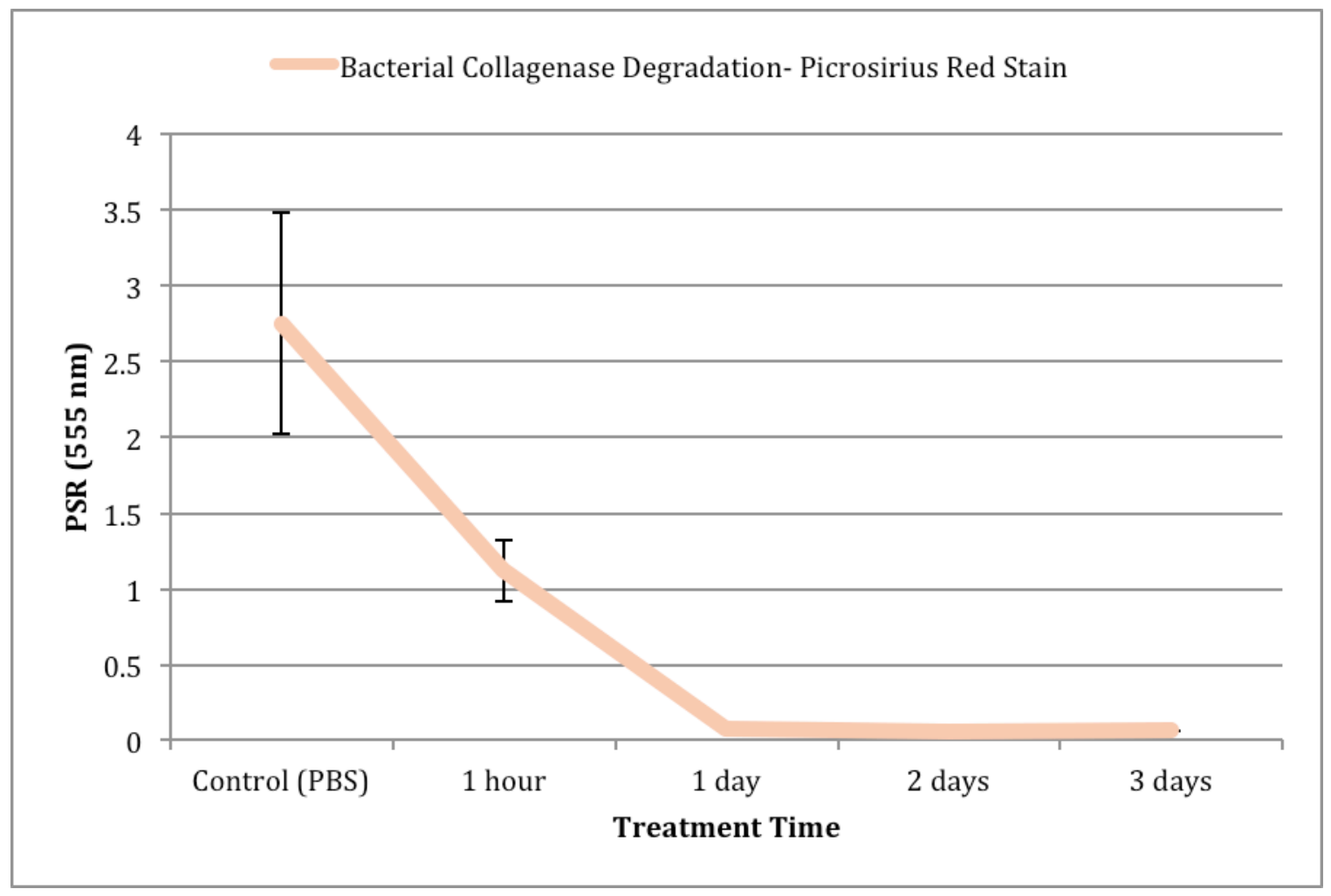

Figure 4. Picrosirius Red Stain. The amount of collagen gel remaining after digestion with $1 \mathrm{mg} / \mathrm{ml}$ bacterial collagenase for 1 hour, 1,2 , and 3 days. 